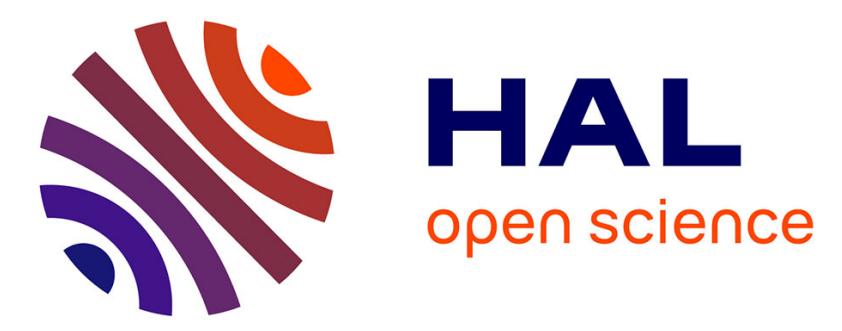

\title{
Time-of-flight mass spectrometry of particle emission during irradiation with slow, highly charged ions
}

L. Skopinski, P. Ernst, M. Herder, R. Kozubek, L. Madauss, S. Sleziona, A.

Maas, N. Königstein, Henning Lebius, A. Wucher, et al.

\section{- To cite this version:}

L. Skopinski, P. Ernst, M. Herder, R. Kozubek, L. Madauss, et al.. Time-of-flight mass spectrometry of particle emission during irradiation with slow, highly charged ions. Review of Scientific Instruments, 2021, 92 (2), pp.023909. 10.1063/5.0025812 . hal-03146416

\section{HAL Id: hal-03146416 \\ https://hal.science/hal-03146416}

Submitted on 19 Feb 2021

HAL is a multi-disciplinary open access archive for the deposit and dissemination of scientific research documents, whether they are published or not. The documents may come from teaching and research institutions in France or abroad, or from public or private research centers.
L'archive ouverte pluridisciplinaire HAL, est destinée au dépôt et à la diffusion de documents scientifiques de niveau recherche, publiés ou non, émanant des établissements d'enseignement et de recherche français ou étrangers, des laboratoires publics ou privés. 


\title{
Time-of-flight mass spectrometry of particle emission during irradiation with slow, highly charged ions
}

\author{
L. Skopinski, ${ }^{1}$ P. Ernst, ${ }^{1}$ M. Herder, ${ }^{1}$ R. Kozubek, ${ }^{1}$ L. Madauß, ${ }^{1}$ S. Sleziona, ${ }^{1}$ A. Maas, ${ }^{1}$ N. Königstein, ${ }^{1}$ H. \\ Lebius, ${ }^{2}$ A. Wucher, ${ }^{1}$ and M. Schleberger ${ }^{1, \text { a) }}$ \\ ${ }^{1)}$ Fakultät für Physik and CENIDE, Universität Duisburg-Essen, 47057 Duisburg, Germany \\ 2) Normandie Univ, ENSICAEN, UNICAEN, CEA, CNRS, CIMAP, 14000 Caen, France
}

(Dated: 28 January 2021)

\begin{abstract}
We describe a setup for the analysis of secondary ions and neutrals emitted from solid surfaces and two-dimensional materials during irradiation with highly charged ions. The ultra-high-vacuum setup consists of an electron beam ion source to produce bunches of ions with various charge states $q$ (e.g. $\mathrm{Xe}^{1+}-\mathrm{Xe}^{46+}$ ) and thus potential energies, a deceleration/acceleration section to tune the kinetic energy of the ions in the range of $5 \mathrm{keV}$ to $20 \mathrm{x} q \mathrm{keV}$, a sample stage for laser-cleaning and positioning of freestanding as well as supported samples, a pulsed excimer laser for postionization of sputtered neutrals, and a reflectron type time-of-flight mass spectrometer enabling us to analyze mass and velocity distributions of the emitted particles. With our setup, contributions from potential and kinetic energy deposition can be studied independently of each other. Charge dependent experiments conducted at a constant kinetic energy show a clear threshold for the emission of secondary ions from $\mathrm{SrTiO}_{3}$. Data taken with the same projectile charge state, but at a different kinetic energy, reveals a difference in the ratio of emitted particles from $\mathrm{MoS}_{2}$. In addition, first results are presented, demonstrating how velocity distributions can be measured with the new setup.
\end{abstract}

\section{INTRODUCTION}

It is well known that the interaction between highly energetic ions and solids leads to the emission of atoms, ions, and electrons, and thus to various kinds of surface modifications. In particular, highly charged ions (HCI) provide a unique opportunity for nanoscaled modifications and thus defect engineering as most of their energy is stored in form of potential energy due to the removal of electrons, which is released upon impact into a nanometric volume. Depending on the material different kind of modifications can be observed after $\mathrm{HCI}$ irradiation $^{1-3}$. For example, for slow HCI surface modifications like pits in $\mathrm{KBr}^{4,5}$ and hillocks in $\mathrm{CaF}_{2}{ }^{6}$, and even craters in $\mathrm{TiO}_{2}{ }^{7}$ and $\mathrm{Si}^{7}$ have been observed. The discovery of twodimensional (2D) materials in $2004^{8}$ sparked new interest in the field as these materials are practically nothing but surface. Hopster et al. were the first to study HCI induced defects in a two-dimensional material and since then this material class has become increasingly popular for defect engineering ${ }^{9}$ and basic research on ion-solid-interactions ${ }^{10}$ alike. For example, defect engineering by ion, and in particular HCI irradiation, may be used to drill pores, which is of particular interest as perforated membranes are envisioned for various applications like DNA sequencing ${ }^{11-13}$ and water desalination ${ }^{14}$. Freestanding 2D samples, on the other hand, have enabled investigations of the HCI surface interaction mechanisms like charge transfer in great detail ${ }^{15-18}$. In those experiments the charge state of HCI transmitted through graphene was analyzed as well as the electron and photon emission taking place during the irradiation.

While the latter experiments provided deep insight into the charge exchange processes they did not yield any information on the target's modification. To investigate this aspect, the

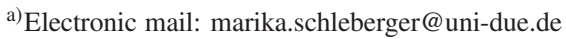

sample had to be taken out of the ultra-high-vacuum (UHV) setup and were analyzed post-mortem in a scanning transmission electron microscope. The same is also true for the abovementioned studies on bulk samples, where also typically postmortem tools have been used to study the defect creation. This means that there is a severe lack of information because direct data on the particles emitted from the target during HCI irradiation is missing. To solve this problem we have set up a UHV-beamline connected to an analysis chamber to obtain complementary information on HCI-induced particle emission, which was hitherto unaccessible. The design of our beamline enables us to vary the kinetic energy of the HCI independent on their charge state (corresponding to their potential energy) in a wide range. For the analysis of emitted secondary ions and neutrals from well-prepared surfaces and 2D materials we make use of time-of-flight (ToF) mass spectrometry in combination with an excimer laser for post-ionization allowing us to obtain mass as well as velocity distributions. In this paper we provide a detailed description of the UHV setup, the associated measurement techniques, and present first results demonstrating the capabilities of our experiment.

\section{EXPERIMENTAL SETUP}

Our setup dedicated to the investigation of highly-chargedion induced collisions at surfaces (called HICS in the following) consists of four major components: i) the ion source, ii) the ion lift for de- and acceleration, the analysis chamber consisting of iii) the sample stage and iv) the mass spectrometer. An overview of the complete setup is given in Fig. 1 and each of the components will be described in detail below. In addition, typical beam parameters are presented as a reference for both, material modification by ion irradiation (Table I) and mass spectrometry (Table II). 


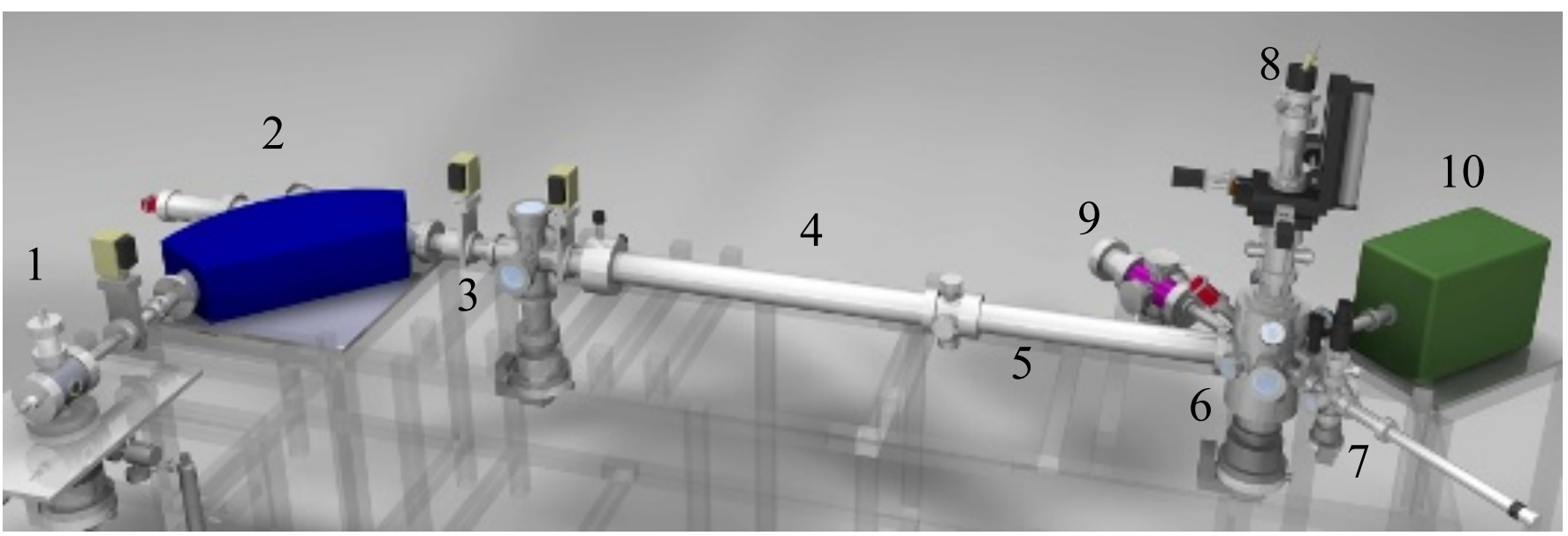

FIG. 1. Schematic view of the HICS setup showing the EBIS ion source (1), bending sector magnet (2), deflection unit (3), ion-lift (4), lens system (5), irradiation chamber (6), vacuum lock (7), manipulator (8), ToF-SIMS (9), and ecximer laser (10).

\section{A. Ion source}

The key element of our setup is an electron beam ion source (Dresden EBIS-A from DREEBIT GmbH, Germany ${ }^{19}$ ) which generates highly charged ions. The operation principle is based on the successive removal of electrons from trapped ions by electron impact ionization. For this purpose, a focused electron beam ionizes gas atoms within three drift tubes. These tubes build a trap for charged particles due to an electric and magnetic potential gradient. The electric potential of the drift tubes $U_{0}$ is typically limited to a range of $3.5 \mathrm{kV}$ to $11 \mathrm{kV}$ with reference to the beamline. The acceleration voltage of the ions $U_{\text {ion }}$ is however given by $U_{\text {ion }}=U_{0}-U_{\mathrm{A}}$ with a typical trap depth of $U_{\mathrm{A}}=180 \mathrm{~V}$. Therefore, the ions exit the source into the beamline (kept at ground potential) with a kinetic energy of $E_{\text {kin }}=q \cdot U_{\text {ion }}$. In general, the operation of the EBIS allows the generation of pulsed ion beams as well as DC beams. For our experiments we typically use pulsed beams which are described in the following. The charge state distribution of ionized atoms is defined by the time spent inside the trap, which is controlled by switching the potential state of the last drift tube (closed: $U_{\mathrm{B} 1}=U_{\mathrm{B} 2}=U_{0}$ and open: $\left.U_{\mathrm{B} 2}<U_{0}\right)$. With the lowering of the last potential $U_{B 2}$, the ion bunch is thus released into the beamline. As projectiles noble gases like argon (up to $q=18+$ ) and xenon (up to $q=46+$ ) are typically used. The base pressure within the ion source is $2.7 \cdot 10^{-10} \mathrm{mbar}$ and the noble gas pressure depends on the chosen charge state, for example $3.5 \cdot 10^{-10}$ mbar for $\mathrm{Xe}^{46+}$ up to $6.5 \cdot 10^{-9} \mathrm{mbar}$ for $\mathrm{Xe}^{2+}$.

A $x$ - and $y$-deflector, as well as an electrostatic lens are used to align the ion beam before a bending sector magnet (Danfysik - bending radius of $90^{\circ}$ ) separates the ions with different charge states contained within the bunch into packages containing exclusively ions with a specific charge $q$ over mass $m$ ratio. By choosing the correct magnetic field strength $B$, only one $q / m$-ratio may pass the magnet under the desired deflection angle of $90^{\circ}$, pass through the aperture and finally enter into the beam line tube. If the extracted $q / \mathrm{m}$ pulse is too
TABLE I. Typical operating parameters for HCI irradiation at the HICS beamline in Duisburg. For selected kinetic and potential energies the ion current of a beam focused to a diameter of $1 \mathrm{~mm}$ is presented.

\begin{tabular}{cccc}
\hline \hline$E_{\text {kin }}[\mathrm{keV}]$ & $E_{\mathrm{pot}}[\mathrm{keV}]$ & ion current $[\mathrm{pA}]$ \\
\hline 5 & 39 & $\left(\mathrm{Xe}^{40+}\right)$ & 0.08 \\
20 & 0.1 & $\left(\mathrm{Xe}^{4+}\right)$ & 2.25 \\
20 & 39 & $\left(\mathrm{Xe}^{40+}\right)$ & 0.1 \\
140 & 2.6 & $\left(\mathrm{Xe}^{16+}\right)$ & 60 \\
200 & 12 & $\left(\mathrm{Xe}^{28+}\right)$ & 12 \\
260 & 12 & $\left(\mathrm{Xe}^{28+}\right)$ & 4 \\
260 & 39 & $\left(\mathrm{Xe}^{40+}\right)$ & 0.8 \\
260 & 59 & $\left(\mathrm{Xe}^{45+}\right)$ & 0.028 \\
400 & 12 & $\left(\mathrm{Xe}^{28+}\right)$ & 16 \\
\hline \hline
\end{tabular}

long in time for a given experiment, a second deflector after the magnet can be used to cut out a temporally defined pulse structure. This well-defined ion pulse can be detected by ion-current measurements realized by a Faraday cup within the irradiation chamber. With the help of a current/voltage amplifier (DHPCA-100 Femto) and a $500 \mathrm{MHz} 2.45 \mathrm{GS} / \mathrm{s}$ oscilloscope (Tektronix DPO 3052), ion-currents of a few fA are measurable.

\section{B. Ion lift}

As outlined in the section before, the acceleration voltage of the ion source is limited to a range of $3.5 \mathrm{keV} \leq U_{\text {ion }} \leq 11 \mathrm{keV}$. This means that, e.g., a $\mathrm{Xe}^{40+}$ ion, with a potential energy of $39 \mathrm{keV}$ would have a kinetic energy of $150-440 \mathrm{keV}$ making it practically impossible to study any effects dominated by the 
TABLE II. Typical parameters for HCI - secondary particle time of flight measurement at the HICS beam line in Duisburg. In recent experiments we used kinetic energies of $5 \mathrm{keV}, 20 \mathrm{keV}$, and $260 \mathrm{keV}$ although faster ions are also possible, see e.g. Fig. 5. All these primary ion bunches have a FWHM of about $1 \mu \mathrm{s}$.

\begin{tabular}{cccc}
\hline \hline$E_{\text {kin }}[\mathrm{keV}]$ & $E_{\text {pot }}[\mathrm{keV}]$ & ions/pulse \\
\hline 5 & 3 & $\left(\mathrm{Xe}^{17+}\right)$ & 2500 \\
5 & 21 & $\left(\mathrm{Xe}^{33+}\right)$ & 680 \\
5 & 39 & $\left(\mathrm{Xe}^{40+}\right)$ & 300 \\
20 & 3 & $\left(\mathrm{Xe}^{17+}\right)$ & 6600 \\
20 & 21 & $\left(\mathrm{Xe}^{33+}\right)$ & 1600 \\
20 & 39 & $\left(\mathrm{Xe}^{40+}\right)$ & 1300 \\
260 & 9 & $\left(\mathrm{Xe}^{26+}\right)$ & 5100 \\
260 & 21 & $\left(\mathrm{Xe}^{33+}\right)$ & 2800 \\
260 & 39 & $\left(\mathrm{Xe}^{40+}\right)$ & 2300 \\
\hline \hline
\end{tabular}

potential energy. Thus, to extend this range, we have implemented a novel deceleration/acceleration system. The most common method to manipulate the kinetic energy of ions is to shift the electrostatic potential of the ion source and the target with respect to each other, so that the ions have to pass a potential gradient. However, this method goes along with serious operating restrictions, because the associated control and measurement devices also need to be shifted to the correct potential, and for security reasons the installation of a Faraday cage is inevitable to screen the high-voltage segment of the setup. To ensure security and keep operation as simple as possible, we have chosen a dynamic method instead, where the beamline is kept at ground potential the whole time. To this end, a $1.5 \mathrm{~m}$ electrically insulated metal tube is mounted inside the vacuum tube, which can be switched to a potential $U_{\text {lift. }}$ As this is a new concept, we will describe the ion lift and its operation in detail in the following paragraphs. The operating principle of deceleration is illustrated in Fig. 2. The ion bunch, represented by the hatched ellipse, is generated in the source at the potential $U_{\text {ion }}$ and thus has a kinetic energy of $E_{\text {kin }}=q \cdot U_{\text {ion }}$ upon leaving the ion source. To modify this energy, the tube of the ion lift is initially grounded but as soon as the complete ion bunch has entered the tube, the potential of the inner tube is rapidly switched to a voltage $U_{\text {lift }}$ via a fast high voltage switch (Behlke HTS 151-03-GSM). As long as the HCI are inside this tube, no force acts upon them. Once they leave the tube however, they have to overcome a potential wall on their way towards the target. To suppress defocusing of the ion bunch when leaving the ion lift due to deceleration, a lens system consisting of ten electrodes was installed to refocus the bunch onto the target. Reaching the target, the remaining kinetic energy is $E_{\text {kin }}=q \cdot\left(U_{\text {ion }}+U_{\text {lift }}\right)$. The sign of the voltage $U_{\text {lift }}$ determines whether the ions are accelerated or decelerated. In our setup $U_{\text {lift }}$ can be tuned from $-10 \mathrm{kV}$ to $+10 \mathrm{kV}$. For higher voltages electrical insulation is no longer sufficient.

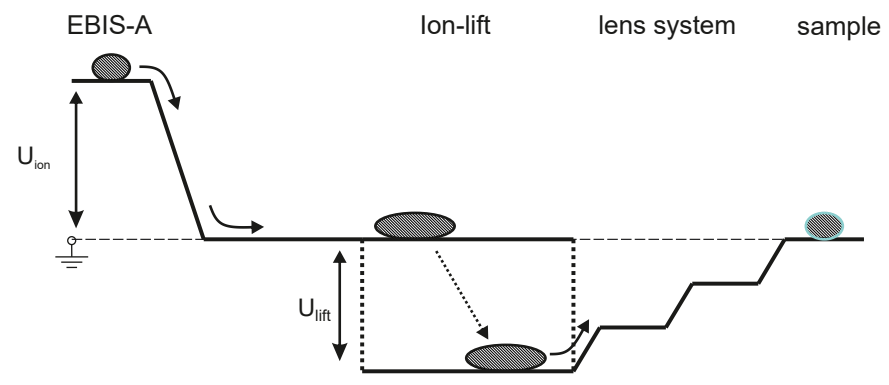

FIG. 2. Schematic presentation of the principle idea of the ion lift ${ }^{20}$. The ion bunch is generated with the potential $U_{\text {ion }}$ and accelerated towards ground potential upon leaving the ion source. Once the complete bunch is within the ion lift tube, the potential is rapidly switched to a negative voltage $U_{\text {lift }}$. When leaving the lift a potential barrier has to be overcome which causes the kinetic energy of the ions to decrease. Along the deceleration path the ions move through a ten-segment lens system to prevent defocusing of the ion bunch. By using a positive $U_{\text {lift }}$ an acceleration of the ions can be realized as well.

To ensure correct operation, the spatial length of the ion bunch has to be smaller than the tube length of the ion lift. Since the ion bunches generated by the EBIS-A do not always meet this criterion, an additional deflector (3) has been implemented into our setup to shorten the length of the ion pulse. This is achieved by switching on a blank voltage $U_{\text {blank }}=500$ $\mathrm{V}$ at a specific time $t_{\text {blank }}$ (with respect to the ion extraction), when the ion bunch passes the deflector. While the front part passes the deflection unit unaffected, the rear part is deflected into the steel chamber of our setup and is therefore no longer available for the experiment. Fig. 3 shows the effect of the deflection unit on the structure of a $\mathrm{Xe}^{40+}$ pulse at a kinetic energy of $E_{\text {kin }}=260 \mathrm{keV}$.

The curves represent the current measurement in the Faraday cup in the irradiation chamber as a function of $t_{\text {flight }}$, which is the time the ions need to arrive at the Faraday cup after they have been extracted from the ion source. Focusing on the original pulse structure, represented by the gray curve in Fig. 3, the first HCI is detected at $t_{\text {flight }} \leq 9 \mu$ s, whereas even at $t_{\text {flight }} \geq 12 \mu$ s ions still reach the Faraday cup as can be seen from a significant ion current. It can therefore be concluded, that the pulse width of the ion bunch is $\geq 3 \mu \mathrm{s}$. With a kinetic energy of $E_{\mathrm{kin}}=260 \mathrm{keV}$ this corresponds to a pulse length of $\approx 1.9 \mathrm{~m}$, which exceeds the length of the ion lift tube by $0.4 \mathrm{~m}$. The set of curves shown in Fig. 3 demonstrates how the structure of the ion bunch changes depending on $t_{\text {blank }}$, which denotes the time when the deflection voltage is activated. Starting at $t_{\mathrm{blank}}=6.6 \mu \mathrm{s}$, one can observe an abrupt decrease of the ion current at $t_{\text {flight }}=11.5 \mu \mathrm{s}$, which corresponds to a chopping of the rear part of the ion bunch. For decreasing $t_{\text {blank }}$, one can see how this abrupt decrease in current shifts towards smaller $t_{\text {flight }}$, while the front part of the ion bunch remains largely unaffected. The pulsed mode of the ion source causes a quick-raising leading edge of the pulse that, compared to the long tail, does not require additional deflecting. As a result of the deflection, the ion bunch length is reduced as intended, but with the obvious drawback, that the 


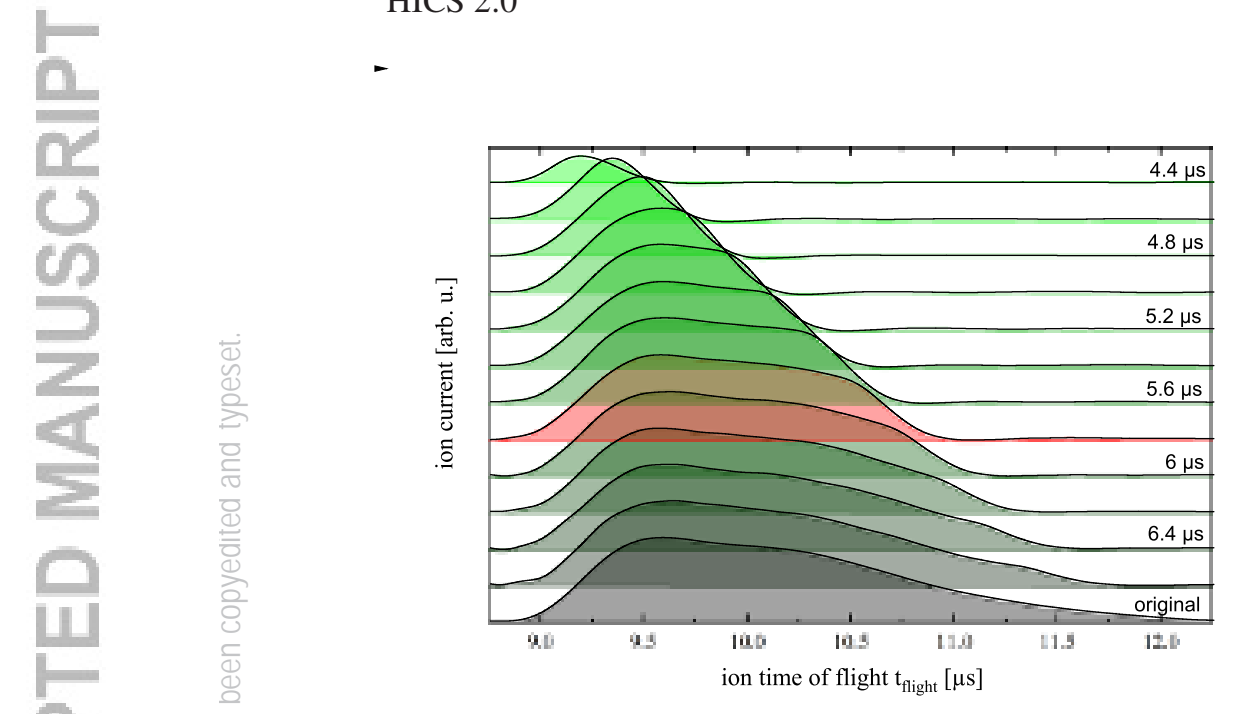

FIG. 3. Ion current measurement in the Faraday cup at the sample stage to analyze the pulse structure of $\mathrm{Xe}^{40+}$ ions with a kinetic energy of $260 \mathrm{keV}^{20}$. Depending on the activation time of the deflecting voltage the pulse length can be tailored. Note that only the difference of $2 \mu$ s in the deflection time can be identified, since the pulse is cut short at the end and therefore the starting time has no significance. In gray the original pulse structure without applying a deflection voltage is shown and the red pulse (at $t_{\text {blank }}=5.8 \mu \mathrm{s}$ ) marks the one used for the demonstration of the ion lift in Fig. 4 and Fig. 5. R. Kozubek, Universitätsbibliothek Duisburg-Essen, 2018; licensed under a Creative Commons Attribution (CC BY) license.

number of ions per ion bunch is reduced. To minimize this effect we use the front part of the pulse which has the highest ion current. A compromise between a short pulse length and a large number of ions per bunch is depicted in red at a blank time of $t_{\text {blank }}=5.8 \mu \mathrm{s}$. For this case, only $20 \%$ of the ions are lost, whereas the pulse length is reduced to a value of around $2 \mu \mathrm{s}$, corresponding to a pulse length of $\approx 1.2 \mathrm{~m}$, which is well below the length of the ion lift. All measurements presented in these following paragraphs were therefore acquired with this value of $t_{\text {blank }}$.

In this paragraph we demonstrate the operation of the ion lift by purposely changing the timing $t_{\text {lift }}$ towards both earlier and later timings with the ion lift switched to a fixed voltage of $U_{\text {lift }}=-4 \mathrm{kV}$. Again, we detect the ion current in the Faraday cup, while changing $t_{\text {lift. }}$. To ensure a good comparability with the measurements regarding the flight duration of the ions, we have set the potentials of the focusing lens system to a constant value. We have chosen the set of voltages such that the ion current is at its maximum for the strongly decelerated ions. For ions of other kinetic energies however, the focusing is not optimal, which leads to a loss of current intensity. For this reason we will focus only on the temporal changes of the ion pulses and omit the discussion of current intensities.

The temporal shape of the ion pulse for $7.4 \mu \mathrm{s} \leq t_{\text {lift }} \leq 9.2$ $\mu \mathrm{s}$ is shown in Fig. $4 \mathrm{c}$ ). For $t_{\text {lift }}>9.2 \mu \mathrm{s}$, shown in black at the bottom of the graph, the lift was switched to a negative voltage after the ion bunch has passed the tube, so that the ion's kinetic energy remains unaffected. As the time $t_{\text {lift }}$ is

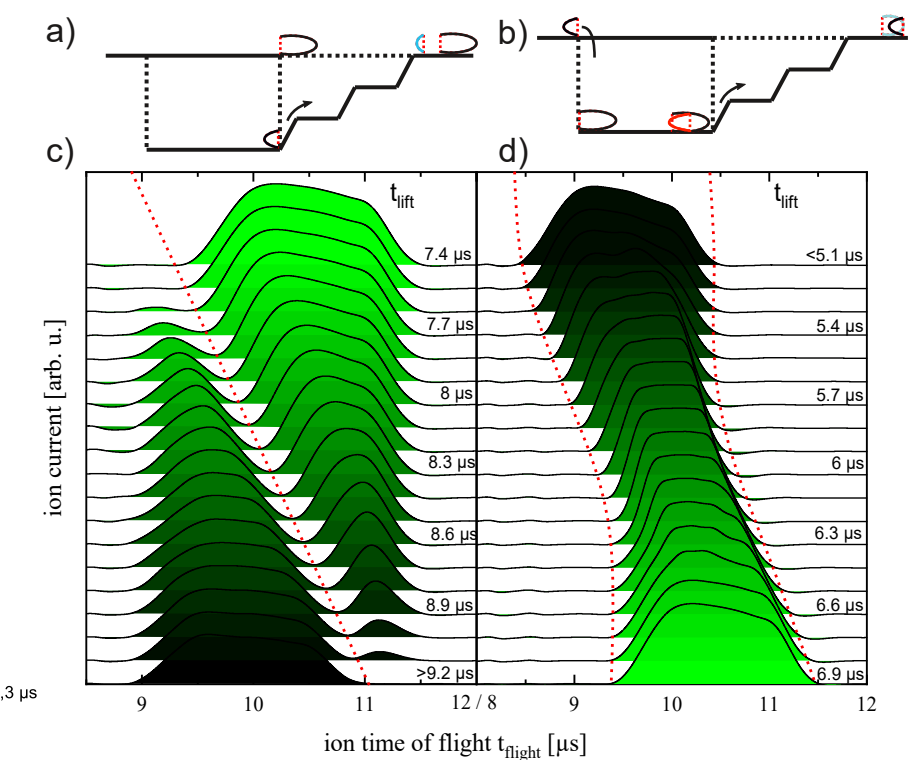

FIG. 4. Ion current measurement of the bunch presented in Fig. 3 after passing the ion lift using a fixed voltage $U_{\text {lift }}=-4 \mathrm{kV}$. Depending on the timing the ion lift cuts the bunch in two parts. In a) and c) the voltage is applied delayed and not all of the ions are decelerated resulting in a splitting of the bunch. In b) and d) only the later part is decelerated as intended, while the first part of the bunch is accelerated due to the negative potential of the ion lift. This leads to a bunching of the ions. Timings between c) and d) $6.9 \mu \mathrm{s} \leq t_{\text {lift }} \leq 7.4$ $\mu$ give the range for optimal operation timings.

reduced, one can observe that the ion bunch is split into two parts at the exit of the ion lift, as illustrated in Fig. 4 a). This is due to the fact that the front part has already left the tube and therefore remains unaffected (ion bunch outlined in black in the sketch) whereas the rear part is still located within the ion lift and as a result is reduced in potential, which consequently leads to a deceleration on the way towards the sample (ion bunch outlined in blue). This manipulation of the kinetic energy of the rear part leads to a delay of these ions and therefore to a clear differentiation between both ion pulse parts in the current measurements as indicated by the dashed red line in Fig. 4 a). For $6.9 \mu \mathrm{s} \leq t_{\text {lift }} \leq 7.4 \mu \mathrm{s}$, the complete ion bunch is located within the lift tube, so that all ions are decelerated and no splitting occurs.

The pulse structure for $5.1 \mu \mathrm{s} \leq t_{\text {lift }} \leq 6.9 \mu$ s is shown in Fig. $4 \mathrm{~d})$. Here once again, the ion bunch is split into two parts. In this case however, the chopping takes place at the entrance of the lift tube as illustrated in Fig. 4 b). Now, the front part of the pulse is located within the ion lift at $t_{\text {lift }}$, whereas the rear part has not yet entered the tube. As the voltage $U_{\text {lift }}$ is applied to the lift tube, the front part is reduced in potential and is decelerated after leaving the ion lift (ion bunch outlined in blue). However, when the front part enters the ion lift, it registers a static negative voltage $U_{\text {lift }}$ at the lift tube, which accelerates the ions. As a consequence, these ions have a higher kinetic energy within the ion lift (ion bunch outlined in red), which is reduced after leaving the lift tube until the ion bunch finally reaches its initial kinetic energy upon hitting 
the target (ion bunch outlined in black). Due to a constant difference in kinetic energy $\Delta E=q \cdot U_{\text {lift }}$ between the front and the rear part along the entire path between the entrance of the ion lift and the target, the rear part of the ion bunch catches up with the front part, which is also observable in the current measurements shown in Fig. $4 \mathrm{~d}$ ). Starting at $t_{\text {lift }}=6.8 \mu$ s one can see a shift of the right flank of the curve towards smaller $t_{\text {flight }}$ with a simultaneous formation of a small additional rise on top of the current curve (best visible for $t_{\text {lift }}=6.6 \mu \mathrm{s}$ at $\left.t_{\text {flight }}=10.4 \mu \mathrm{s}\right)$. This is the aforementioned rear part of the ion bunch which catches up with the front part. For decreasing $t_{\text {lift }}$, the number of ions located within the ion lift during switching of the lift tube decreases until finally at $t_{\text {lift }}<5.1 \mu \mathrm{s}$ the complete ion bunch is located in front of the lift.

These results demonstrate that the correct operation of the ion lift requires an optimal timing of the switching. This optimum can be inferred from the measurements presented above to be in the range of $6.9 \mu \mathrm{s} \leq t_{\text {lift }} \leq 7.4 \mu \mathrm{s}$. With this optimized timing scheme we can now proceed to demonstrate that the ion lift can be used to deliver pulses of highly charged ions with a given charge state and with variable kinetic energy. As an example we present data from a $\mathrm{Xe}^{40+}$ ion pulse with an initial kinetic energy of $260 \mathrm{keV}$ (gray data in Fig 5). The ion lift switching time was set to $t_{\text {lift }}=6.9 \mu$ s and the lift voltage was varied in the range of $-6 \mathrm{kV} \leq U_{\text {lift }} \leq 6 \mathrm{kV}$, resulting in a deceleration (blue curves) down to $45 \mathrm{keV}$ and acceleration (red curves) up to $475 \mathrm{keV}$, respectively. For all parameters, the complete pulse is affected and a sufficiently large ion current can be measured in the Faraday cup at the sample stage. Note, that the arrival time of the pulse shifts and changes in its temporal shape occur on a timescale of $\mu \mathrm{s}$. For experiments accessing data on similar timescales these effect have to be taken into account as will be discussed below.

\section{Sample stage}

Within the irradiation chamber a sample stage and a Faraday cup (FC) are mounted on a five-axis manipulator (VG Scienta) movable in all three directions and rotatable around two axes. The Faraday cup is an important feature of the setup because for a quantitative analysis the measured signal has to be normalized to the number of primary ions. To this end, the Faraday cup is covered by two metal apertures. The top one is on ground potential and has a circular opening with a diameter of $1 \mathrm{~mm}$, into which the ion beam is focused. The second one directly above the cup has a slightly bigger opening and is on negative potential to prevent secondary electrons from leaving the cup. In this way, only electrons needed to neutralize the HCIs are detected in the cup.

As sample holders we use modified Omicron holders that allow the addition of apertures with various openings and a sample size of $9 \times 9 \mathrm{~mm}^{2}$ with a thickness of up to $2 \mathrm{~mm}$. Since the sample is always attached directly to the aperture, the sample surface position is very well defined. Exact positioning of the sample is important for the time of flight mass spectrometry and to ensure this, we have two cameras viewing the sample under different angles (colored red in Fig. 1).

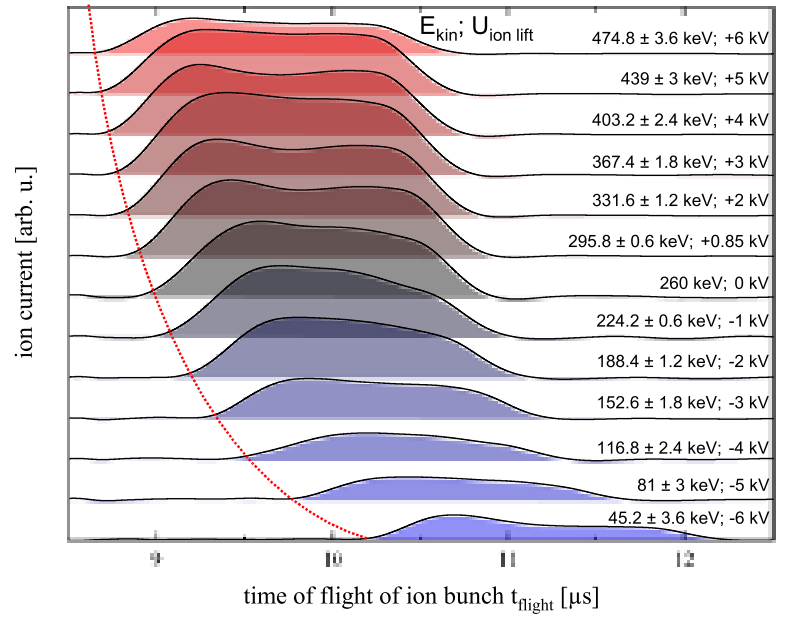

FIG. 5. Ion current measurement as a function of the time of flight of the ion bunch for various ion lift potentials. In gray with a kinetic energy of $260 \mathrm{keV}$ the pulse structure is again presented. For increasing potential $U_{\text {lift }}$ a slight shift of the bunch towards smaller times of flight can be observed (red). In blue, the decelerated bunches are shown which are strongly shifted towards longer times of flight. This non-linear shift results from the $E^{-1 / 2}$ dependence of the time of flight on the kinetic energy.

One is positioned at the irradiation chamber (6) and the second behind the magnet (2) looking through the beamline onto the sample. In addition, a red-light alignment laser is pointing through the spectrometer onto the sample. The time of flight mass spectrometry measurements have to be conducted with the sample tilted by $45^{\circ}$ with respect to the ion beam. To ensure correct current measurements despite this geometrical limitation we have two options. We can either use an aperture for the FC with an elliptical opening, the size of which is such that when turned the same circular section of the beam hits the sample as detected by the cup. As an alternative, a new manipulator head was designed as shown in Fig. 6. The left drawing gives an overview of the manipulator head with two FCs - with again two apertures each - on the top and the sample positioned on the electrically insulated bottom. The two FCs allow to measure the ion current both under $90^{\circ}$ and $45^{\circ}$ with respect to the HCI beam as shown in the middle of Fig. 6 . Also, the sample position has an opening at the back allowing the investigation of freestanding samples (mounted on TEM grids) without any contribution from backscattered particles. Note also that FCs and sample apertures are mounted in the same plane enabling us to switch between both positions with minimal movement of the manipulator.

For cleaning sample surfaces our setup is equipped with an ion gun for $\mathrm{Ar}^{+}$sputtering and a heating stage. In addition to the conventional cleaning procedure by sputtering and heating, we have equipped our setup with a $445 \mathrm{~nm}$ high power diode laser $^{21}$ to implement a laser cleaning process. Good results with graphene ${ }^{21,22}$ and very promising first tests with single layer $\mathrm{MoS}_{2}$ in our lab confirm that laser cleaning is an excellent and time saving alternative in particular for - but not 


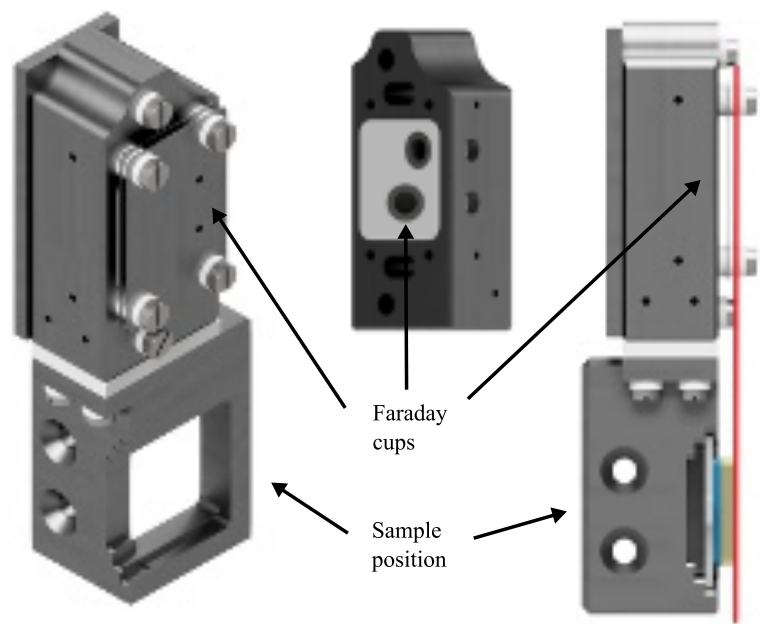

FIG. 6. New design of the manipulator head. The top part includes two Faraday cups to measure the ion current under both $90^{\circ}$ and $45^{\circ}$ with respect to the ion beam. The sample position on the bottom part is electrically insulated from the rest of the manipulator and has an opening in the back.

limited to - 2D materials in UHV setups.

\section{Time-of-flight mass spectrometer}

For the analysis of sputtered neutral and ionized particles, a home-built reflectron time-of-flight (ToF) mass spectrometer, described in detail elsewhere ${ }^{23,24}$, was mounted to the analysis chamber. The ToF spectrometer is positioned at $45^{\circ}$ with respect to the incident HCI beam. During a measurement, the sample is positioned orthogonally to the ion optical axis of the spectrometer so that the projectiles hit the sample at $45^{\circ}$ with respect to the sample surface normal. Ionized particles emitted from the surface are extracted by pulsing the sample potential from ground to $1250 \mathrm{~V}$ using a very fast HV switch (Behlke HTS31-03-GSM) with a rise time of about 21 ns. With our setup not only the positive or negative ionized emitted particles can be detected (Secondary Ion Mass Spectrometry - SIMS), but also the neutral part of emitted particles can be post-ionized by a pulsed $157 \mathrm{~nm}$ excimer laser (Coherent ExciStar XS 500), and analyzed subsequently (Secondary Neutral Mass Spectrometry - SNMS). The laser is operated with a frequency up to $500 \mathrm{~Hz}$ and has a maximum pulse energy of $3 \mathrm{~mJ}$. Using a $\mathrm{CaF}_{2}$ lens the laser beam is aligned 1 $\mathrm{mm}$ above and parallel to the surface and focused to a spot diameter of about $0.5 \mathrm{~mm}$. Ionized particles which enter the ToF spectrometer are detected by a dual microchannel plate (MCP) in chevron configuration, operated at $2000 \mathrm{~V}$. The front of the stack is kept on ground potential to ensure that positive and negative ions hit the detector with the same kinetic energy of about $1.2 \mathrm{keV}$. The MCP signal is digitized using a Signatec PDA 1000 digitizer board. This type of mass spectrometer allows simultaneous investigation of small mass particles as well as large organic molecules with mass up to several thou-

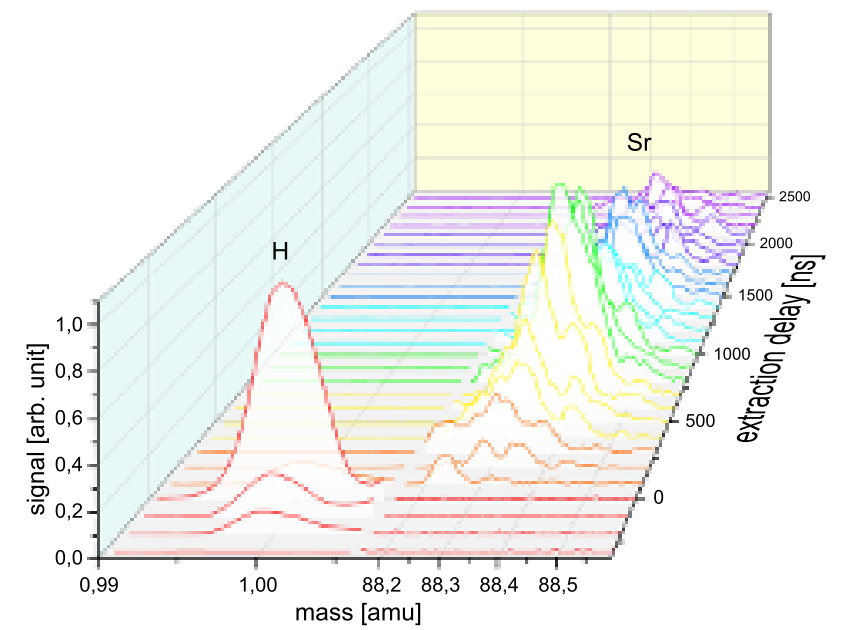

FIG. 7. Measured hydrogen SIMS and strontium SNMS peak resulting from irradiation of $\mathrm{SrTiO}_{3}(100)$ with $\mathrm{Xe}^{40+}$ ions shown as a function of the extraction delay. The hydrogen peak reaches its maximum signal at earlier extraction delays than the heavier strontium peak.

sand atomic mass units. Ions which start at different distances from the sample surface, can be focused to a sharp time peak with a mass resolution $(\mathrm{m} / \Delta m)$ of about 500 due to the reflection inside the ToF spectrometer.

The ToF measurements are performed with pulsed extraction and the mass spectra are acquired at different extraction delays relative to the primary ion pulse impinging on the surface. This delayed extraction is necessary because the emitted particles need time to reach the laser volume to be ionized before they can be extracted by the sample potential. The volume above the sample from which ions can contribute to the signal will be called sensitive volume in the following. The mass spectra are acquired at different extraction delays to collect particles with different emission velocities due to either the mass of a particle or its emission energy.

This is shown in Fig. 7, where the hydrogen ( $m=1 \mathrm{amu})$ peak as the first signal we detect in each SNMS spectrum and the strontium ( $m=88 \mathrm{amu})$ peak as a heavier particle that represents the sample, are presented. The spectra were measured during irradiation of single-crystalline $\mathrm{SrTiO}_{3}(100)$ with $\mathrm{Xe}^{40+}$ ions with a kinetic energy of $260 \mathrm{keV}$ as a function of the extraction delay. To optimize the signal-to-noise ratio, each spectrum was measured with 250 repetitions and the average signal has been evaluated. The first cycle was collected before the primary ion beam hit the surface. Then, the extraction delay has been shifted in steps of $100 \mathrm{~ns}$ towards longer delays until the last cycle has been collected $2.5 \mu$ s after the first primary ions hit the surface.

The hydrogen signal has its maximum intensity at an extraction delay of $0 \mathrm{~ns}$ while the strontium reaches its maximum intensity after $800 \mathrm{~ns}$. As expected, the heavier strontium particles reach the sensitive volume of the spectrometer at later extraction delays than the hydrogen particles. However, not only the position of the maximum signal differs, but 


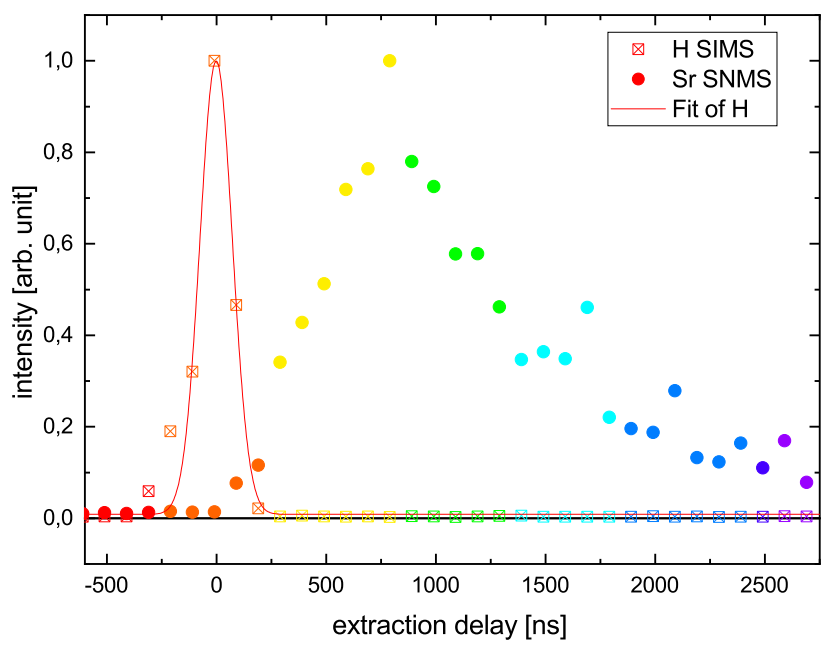

FIG. 8. Determined intensity of the hydrogen SIMS and strontium SNMS peaks presented in Fig. 7 in dependence of the extraction delay of the respective spectra. Each dot gives the integrated intensity of one peak with the same color code as in Fig. 7. The Gaussian fit of the $\mathrm{H}$ intensity gives a rough estimate of the primary $\mathrm{HCI}$ pulse.

also the time span during which its signal is present in the spectra.

The plot in Fig. 7 can be simplified by integrating the signal over a specific mass. This area is then proportional to the number of particles of a given species which were detected with the MCP. This is demonstrated in Fig. 8, where the intensity is shown as a function of the extraction delay. The $\mathrm{H}$ and $\mathrm{Sr}$ peak of each spectrum presented in Fig. 7 have been integrated to determine the intensity. The same color code as in the previous figure identifies each peak. The full width at half maximum of the hydrogen peaks (here $200 \mathrm{~ns}$ ) is a good indicator for the width of the primary ion pulse. The flight time of a hydrogen atom with a kinetic energy of $1 \mathrm{eV}$ across the distance of $1 \mathrm{~mm}$ is around $72 \mathrm{~ns}$. As the lightest detected particle in the absence of electrons it is therefore also used to determine the zero point for the flight time $t_{\mathrm{f}}$ of neutral particles. This method to determine the zero point is compared to electron measurements with the Faraday cup and discussed in detail by Herder et al. ${ }^{25}$. Note that because the stage delay steps of $100 \mathrm{~ns}$ are very big compared to the width of the $\mathrm{H}$ signal of $200 \mathrm{~ns}$, the zero time cannot be determined without a significant error. Therefore, we abstain from deriving a velocity distribution suitable for a detailed analysis from this exemplary data, but it may still serve as an example for the principle, as we detect a general change in the flight time distribution. For example, an increased intensity at later extraction delays means that there are more particles with a low velocity even if we cannot determine their exact kinetic energy.

The conversion of the flight time distribution $S\left(t_{\mathrm{f}}\right)$ into a velocity distribution $f(v)$ via the transformation $f(v) \propto S\left(t_{\mathrm{f}}\right) \times t_{\mathrm{f}}$ has been described before ${ }^{26,27}$. To obtain data suitable for a quantitative analysis of the velocity distribution, several parameters can be optimized as e.g. the distance between the

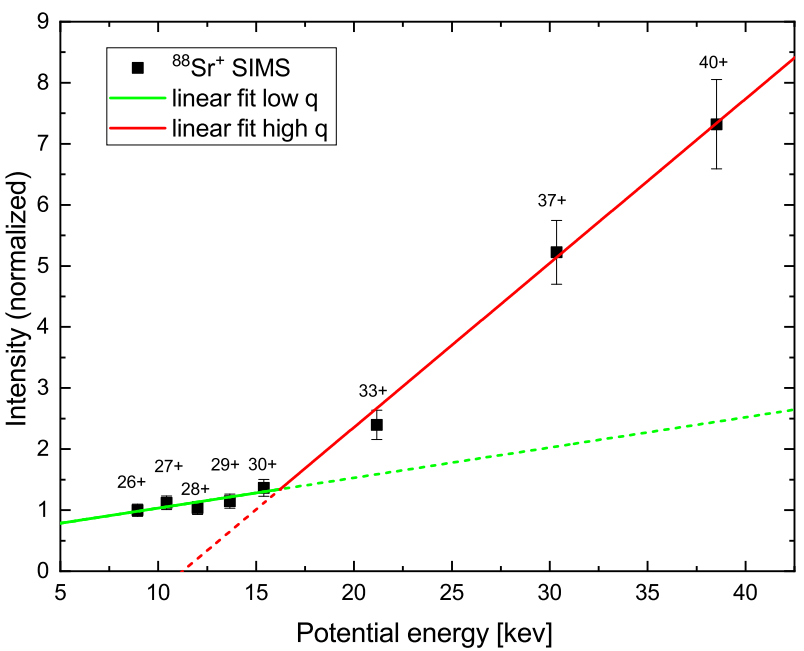

FIG. 9. Signal yield of ${ }^{88} \mathrm{Sr}^{+}$secondary ions per primary ion as a function of the potential energy of the primary ion. Error bars indicate the error in the measurement of the HCI current.

sample surface and the laser pulse used for the post ionization (see Ref. ${ }^{25}$ ) and the duration of the primary ion pulse which can be achieved with our deflection unit as described above (see Table II). However, both measures lead to a significant signal decrease and must therefore be carefully balanced. Also, it is advantageous to investigate heavier species, because the error of the primary ion pulse width is less influential at lower velocities or larger delays.

\section{RESULTS}

In the final section we present first results obtained with our HICS setup. As shown above, we can select the potential and kinetic energy of the projectiles independently of each other and we will discuss basically two sets of data, one obtained with a variable potential energy and at fixed kinetic energy and a second one obtained with two different kinetic energies but identical charge states. Finally, we present as first result a velocity distribution of particles emitted from a single layer of $\mathrm{MoS}_{2}$.

We begin with SIMS data obtained from a single crystal of $\mathrm{SrTiO}_{3}$ irradiated with Xe ions at a fixed kinetic energy of $260 \mathrm{keV}$ with charge states ranging from $26+$ to $40+$. For each charge state a primary ion pulse of $2 \mu$ s was used. The area under each peak corresponding to ${ }^{88} \mathrm{Sr}^{+}$ions was summed up, divided by the number of repetitions, and primary ions per pulse. In Fig. 9 the normalized (with respect to the primary ion current) intensity of ${ }^{88} \mathrm{Sr}^{+}$ions emitted during irradiation is shown as a function of the potential energy of the primary ions.

The normalized intensity of the strontium ions sputtered by $\mathrm{Xe}$-ions with a potential energy between 8 and $15 \mathrm{keV}$ is almost constant (the slope of the green line is $0.05 \mathrm{keV}^{-1}$ ) at a value of 1 . In contrast, the intensity of ${ }^{88} \mathrm{Sr}^{+}$ions sputtered by $\mathrm{Xe}$-ions with higher charge states increases significantly 
and reaches a value as high as 7.3 for $\mathrm{Xe}^{40+}$. Using a linear fit to evaluate the higher charge states, a slope of $0.27 \mathrm{keV}^{-1}$ has been determined. From the different slopes in Fig. 9 one can derive a threshold of $16.1 \mathrm{keV}$ beyond which the potential energy has a significant influence on the sputter yields of $\mathrm{Sr}^{+}$ ions. This is in agreement with results from El-Said et al. ${ }^{28}$ obtained by scanning force microscopy on irradiated $\mathrm{SrTiO}_{3}$. Based on the irradiation with HCI $(q=28-37)$ of a kinetic energy of $4.5 \cdot q \mathrm{keV}$, their work predicts a potential energy threshold for nano-hillock formation between $15.4 \mathrm{keV}$ and $30.4 \mathrm{keV}$. Our results demonstrate that first of all, both the increase in particle emission and the formation of nano-hillocks exhibit the same potential energy threshold. This leads to the important conclusion that the formation of nano-hillocks on $\mathrm{SrTiO}_{3}$ is therefore always accompanied by the emission of particles. Second, the good agreement with SFM measurements validates the new method presented here. In contrast to SFM, it is much quicker, more sensitive and is operated inoperando, which makes it a useful and efficient tool to determine the dependence of the defect mechanism on the primary ion energy.

As a second example we present data obtained with a fixed charge state and with variable kinetic energy. For these experiments a molybdenum disulphide $\left(\mathrm{MoS}_{2}\right)$ bulk crystal has been prepared by removing the upper layer with $3 \mathrm{M}$ tape. This procedure is known to yield a clean, atomically flat surface and therefore no further cleaning has been done in-situ. After introducing the sample into the chamber via the load-lock, SNMS-spectra with different extraction timings were taken during the irradiation with $\mathrm{Xe}^{40+}$ ions $\left(\mathrm{E}_{\text {pot }}=38.5 \mathrm{keV}\right)$ with a kinetic energy of $5 \mathrm{keV}$ and $260 \mathrm{keV}$, respectively. In Fig. 10 the sums of all SNMS spectra taken for the two cases are shown. Because it is difficult to derive absolute yields from SNMS/SIMS spectra, we will discuss in the following only the ratios of mass peaks obtained with different ion beams. To further simplify comparisons of these ratios, the sum spectra have been normalized to the molybdenum signal.

Both mass spectra clearly show the typical pattern of $\mathrm{MoS}_{2}$ which consists of the molybdenum isotopes at masses 92 $100 \mathrm{amu}$ and the sulphur $\mathrm{S}_{2}$ clusters at masses $64 \mathrm{amu}$. Note, that typically no mono-atomic sulphur signal is detected. The more prominent peaks appearing at lower masses can be attributed to hydrogen, carbon, and their compounds, which are typical contaminations. Due to their high sputter probability these contaminants are easily detected, and their apparent abundance should not be misinterpreted as a surface completely covered with adsorbates. However these data show, that obtaining a sufficiently clean surface remains an issue to be solved.

When comparing the spectra obtained with the slow $(5 \mathrm{keV})$ $\mathrm{HCI}$ with the ones obtained with $\mathrm{HCI}$ at $260 \mathrm{keV}$, the most obvious feature is the increase of the peaks stemming from contaminations. These elements are likely to occur only at the surface, which tells us that the sensitivity towards surface contaminations is apparently higher for slow projectiles. From the ratios alone we can however not determine whether only the signal from these adsorbates has increased or whether the molybdenum signal (to which the spectra are normalized)

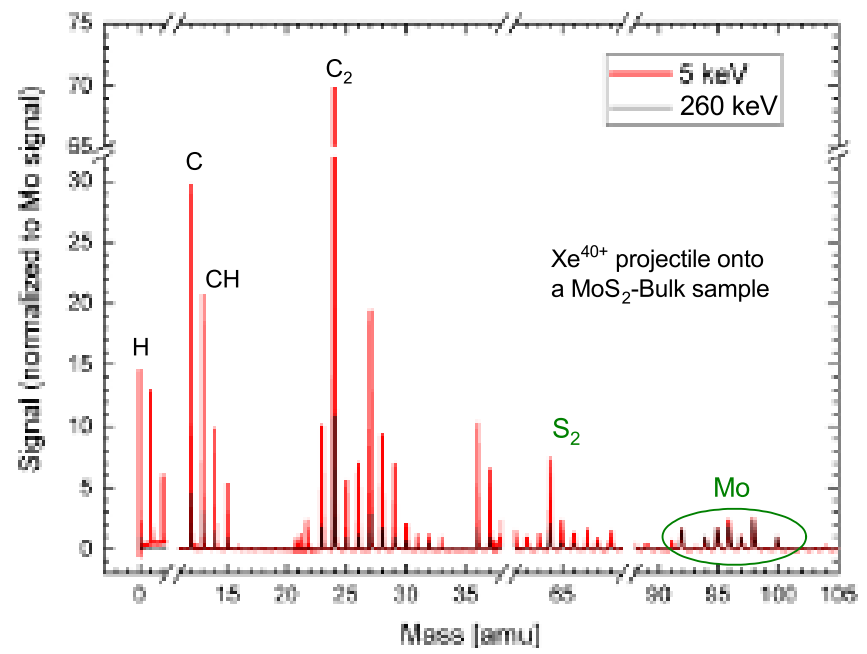

FIG. 10. SNMS spectra of bulk $\mathrm{MoS}_{2}$ irradiated with $\mathrm{Xe}^{40+}$ projectiles with a kinetic energy of $5 \mathrm{keV}$ and $260 \mathrm{keV}$, respectively, each normalized to the Mo signal. The graph shows the increased surface sensitivity of the slow projectile - through increased signal from adsorbates - as well as a changed ratio between the $\mathrm{S}_{2}$-cluster and the Mo-particles.

has decreased. In addition, the beam of slow ions is not as well focused and therefore may also sputter some additional contaminations from the aperture which is mounted directly above the sample (see section II.C.). This latter uncertainty is typical for the ubiquitous carbohydrates but is negligible for sulfur which is absent in the aperture. Our data reveals that the ratio of the sulphur peaks is significantly enhanced by a factor of 3.4 for the slower HCI as well. The basal plane of $\mathrm{MoS}_{2}$ is terminated by a layer of sulphur atoms and it is therefore very likely that indeed the SNMS signal for slower projectiles originates pre-dominantly from the uppermost layers while the faster projectiles generate particle emission mostly from deeper layers.

Our findings are perfectly in line with simulations by Lemell et al. on HCI irradiation of $\mathrm{CaF}_{2}{ }^{29}$. They have simulated the energy deposition into the target based on electron transport and showed that temperatures are generated at the impact site which are sufficiently high to drive a solid-liquid phase transition. The extension and in particular the depth of the affected volume depend strongly on the kinetic energy of the projectile. The deposition depth is basically limited to a very surface near region (ca. $1 \mathrm{~nm}$ ) for slow highly charged ions. However, while this suggests that the primary mechanism for particle emission due to HCI irradiation is related to this phase transition, the mechanism is, in fact, still under discussion. In order to test if the emission is indeed related to a temperature increase, we use our setup to look into the velocity distribution of the emitted particles.

As our final example, we present data from an SNMSmeasurement on $2 \mathrm{D} \mathrm{MoS} 2$ irradiated with $\mathrm{Xe}^{q+}$ primary ions with a kinetic energy of $5 \mathrm{keV}$ in Fig. 11. Both graphs are normalized to the primary ion count and therefore, the two charge states can be easily compared. The intensity in a) is 

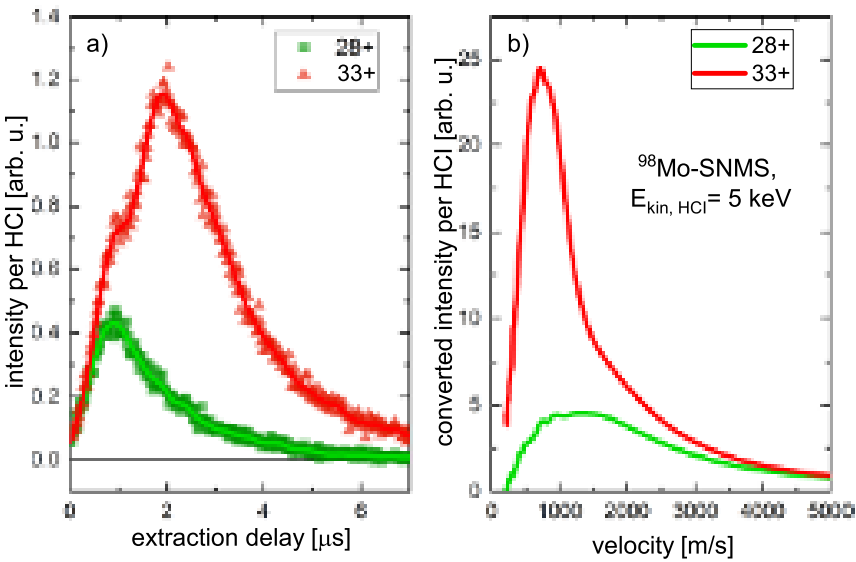

FIG. 11. a) Determined signal per $\mathrm{HCI}$ by $\mathrm{Xe}^{28+}$ and $\mathrm{Xe}^{33+}$ primary ions in dependence of the extraction delay of the measured spectra Each dot gives the integrated intensity of one ${ }^{98}$ Mo peak while the lines show the smoothed development of the signal. The data converted into a velocity distribution is shown in b). Both graphs show an increase in slow particles for the higher charge state.

determined by integrating over the peak of the ${ }^{98}$ Mo isotope for each extraction delay. As described above, we used the hydrogen signal to determine the zero point of the extraction delay. Already in this visualization the difference between the two charge states $28+$ and $33+$ is apparent as there seems to be a second contribution for the higher charge state at later extraction delays. In Fig. 11 b) we present the result of the conversion of this data into a velocity distribution. The previous observation of a second contribution due to the higher charge of the projectile can again be clearly identified. The green curve $(28+)$ has its maximum at around $1500 \mathrm{~m} / \mathrm{s}$ while the red curve (33+) only shows a shoulder at this velocity. Most of the particles emitted due to the irradiation with the $33+$ primary HCI have a velocity of less than $1000 \mathrm{~m} / \mathrm{s}$, which would correspond to a kinetic energy of around $0.16 \mathrm{eV}$. A similar experiment was recently performed by Herder et al. with our setup using a clean indium surface as target ${ }^{25}$. The irradiation was performed with $\mathrm{Xe}^{q+}$ ions with different charge states at a fixed kinetic energy of $20 \mathrm{keV}$. Also there a striking contribution of very slow particles for higher projectile charge states was observed. These at first glance counter-intuitive results demonstrate the potential of our setup to shed new light on the ion-solid interaction mechanisms.

\section{SUMMARY AND OUTLOOK}

We have presented the design and operating principle of our HICS setup to investigate the interaction of HCI with surfaces by means of time-of-flight secondary ion and neutral mass spectrometry. The key feature of the beamline is the ion lift enabling us to de- and accelerate HCI after their extraction from the ion source. The improved compatibility of the stage with freestanding samples and the method of laser cleaning paves the way to promising studies of $2 \mathrm{D}$ materials and their novel applications.

The full control over the ion pulse length in combination with the post-ionization and the delayed extraction scheme offers the unique possibility to study velocity distributions of HCI-induced particle emission. The independent control of kinetic and potential energy, respectively, allows us to investigate the different interaction mechanisms and to establish threshold values. Furthermore, with respect to defect engineering, irradiation parameters can be optimized for example for either maximum efficiency or minimum damage.

\section{DATA AVAILABILITY STATEMENT}

The data that support the findings of this study are available from the corresponding author upon reasonable request.

\section{ACKNOWLEDGMENTS}

We acknowledge support from the DFG by funding SCHL 384/20-1 (project number 406129719) and project C5 within the SFB1242 "Non-Equilibrium Dynamics of Condensed Matter in the Time Domain" (project number 278162697).

${ }^{1}$ A. Arnau, F. Aumayr, P. M. Echenique, M. Grether, W. Heiland, J. Limburg, R. Morgenstern, P. Roncin, S. Schippers, R. Schuch, N. Stolterfoht, P. Varga, T. Zouros, and H. P. Winter, "Interaction of slow multicharged ions with solid surfaces," Surf. Sci. Rep 27, 113-239 (1997).

${ }^{2}$ F. Aumayr and H. Winter, "Potential sputtering," Philos. Trans. R. Soc. A 362, 77-102 (2004).

${ }^{3}$ F. Aumayr, S. Facsko, A. S. El-Said, C. Trautmann, and M. Schleberger, "Single ion induced surface nanostructures: a comparison between slow highly charged and swift heavy ions," J. Phys. Condens. Materie 23, 393001 (2011).

${ }^{4}$ S. Facsko, R. Heller, R. A. Wilhelm, and W. Möller, "Nanostructures formed on $\mathrm{KBr}$ surfaces by the impact of highly charged ions," J. Phys. Conf. Ser 194, 132022 (2009).

${ }^{5}$ R. Heller, S. Facsko, R. A. Wilhelm, and W. Möller, "Defect mediated desorption of the $\mathrm{KBr}(001)$ surface induced by single highly charged ion impact," Physical Review Letters 101, 096102 (2008).

${ }^{6}$ A. S. El-Said, R. Heller, W. Meissl, R. Ritter, S. Facsko, C. Lemell, B. Solleder, I. C. Gebeshuber, G. Betz, M. Toulemonde, W. Möller, J. Burgdörfer, and F. Aumayr, "Creation of nanohillocks on $\mathrm{CaF}_{2}$ surfaces by single slow highly charged ions," Physical Review Letters 100, 237601 (2008).

${ }^{7}$ M. Tona, Y. Fujita, C. Yamada, and S. Ohtani, "Electronic interaction of individual slow highly charged ions with $\mathrm{TiO}_{2}(110)$," Phys. Rev. B 77, 242 (2008).

${ }^{8}$ K. S. Novoselov, D. Jiang, F. Schedin, T. J. Booth, V. V. Khotkevich, S. V. Morozov, and A. K. Geim, "Two-dimensional atomic crystals," Proc. Natl. Acad. Sci. U.S.A. 102, 10451-10453 (2005).

${ }^{9}$ M. Schleberger and J. Kotakoski, "2D Material Science: Defect Engineering by Particle Irradiation,” Materials 11 (2018), 10.3390/ma11101885.

${ }^{10}$ F. Aumayr, K. Ueda, E. Sokell, S. Schippers, H. Sadeghpour, F. Merkt, T. F. Gallagher, F. B. Dunning, P. Scheier, O. Echt, T. Kirchner, S. Fritzsche, A. Surzhykov, X. Ma, R. Rivarola, O. Fojon, L. Tribedi, E. Lamour, J. R. Crespo López-Urrutia, Y. A. Litvinov, V. Shabaev, H. Cederquist, H. Zettergren, M. Schleberger, R. A. Wilhelm, T. Azuma, P. Boduch, H. T. Schmidt, and T. Stöhlker, "Roadmap on photonic, electronic and atomic collision physics: III. Heavy particles: with zero to relativistic speeds," J. Phys. B 52, 171003 (2019). 
${ }^{11}$ S. Garaj, W. Hubbard, A. Reina, J. Kong, D. Branton, and J. A Golovchenko, "Graphene as a subnanometre trans-electrode membrane," Nature 467, 190-193 (2010).

${ }^{12}$ Z. Gu, Y. Zhang, B. Luan, and R. Zhou, "DNA translocation through single-layer boron nitride nanopores," Soft Matter 12, 817-823 (2016).

${ }^{13}$ J. Feng, K. Liu, R. D. Bulushev, S. Khlybov, D. Dumcenco, A. Kis, and A. Radenovic, "Identification of single nucleotides in $\mathrm{MoS}_{2}$ nanopores," Nat. Nanotechnol. 10, 1070-1076 (2015).

${ }^{14}$ M. Heiranian, A. B. Farimani, and N. R. Aluru, "Water desalination with a single-layer $\mathrm{MoS}_{2}$ nanopore," Nat. Commun. 6, 8616 (2015).

${ }^{15}$ E. Gruber, R. A. Wilhelm, R. Pétuya, V. Smejkal, R. Kozubek, A. Hierzenberger, B. C. Bayer, I. Aldazabal, A. K. Kazansky, F. Libisch, A. V. Krasheninnikov, M. Schleberger, S. Facsko, A. G. Borisov, A. Arnau, and F. Aumayr, "Ultrafast electronic response of graphene to a strong and localized electric field," Nat. Commun. 7, 13948 (2016).

${ }^{16}$ R. A. Wilhelm, E. Gruber, J. Schwestka, R. Kozubek, T. I. Madeira, J. P. Marques, J. Kobus, A. V. Krasheninnikov, M. Schleberger, and F. Aumayr, "Interatomic Coulombic Decay: The Mechanism for Rapid Deexcitation of Hollow Atoms," Physical Review Letters 119, 103401 (2017).

${ }^{17}$ J. Schwestka, A. Niggas, S. Creutzburg, R. Kozubek, R. Heller, M. Schleberger, R. A. Wilhelm, and F. Aumayr, "Charge-Exchange-Driven LowEnergy Electron Splash Induced by Heavy Ion Impact on Condensed Matter," J. Phys. Chem. Lett. 10, 4805-4811 (2019).

${ }^{18}$ S. Creutzburg, J. Schwestka, A. Niggas, H. Inani, M. Tripathi, A. George, R. Heller, R. Kozubek, L. Madauß, N. McEvoy, S. Facsko, J. Kotakoski, M. Schleberger, A. Turchanin, P. L. Grande, F. Aumayr, and R. A. Wilhelm, "Vanishing influence of the band gap on the charge exchange of slow highly charged ions in freestanding single-layer $\mathrm{MoS}_{2}$," Physical Review B 102 (2020), 10.1103/PhysRevB.102.045408.

${ }^{19}$ G. Zschornack, M. Kreller, V. P. Ovsyannikov, F. Grossman, U. Kentsch, M. Schmidt, F. Ullmann, and R. Heller, "Compact electron beam ion sources/traps: review and prospects,” Rev. Sci. Instrum 79, 02 A703 (2008)

${ }^{20} \mathrm{R}$. Kozubek, Analyse von Defektstrukturen in zweidimensionalen Materialien nach der Interaktion mit hochgeladenen Ionen (Universitätsbibliothek Duisburg-Essen, Duisburg and Essen, 2018).

${ }^{21}$ M. Tripathi, A. Mittelberger, K. Mustonen, C. Mangler, J. Kotakoski, J. C. Meyer, and T. Susi, "Cleaning graphene: Comparing heat treatments in air and in vacuum," Phys. Status Solidi Rapid Res. Lett. 11, 1700124 (2017).

${ }^{22}$ O. Ochedowski, B. K. Bußmann, and M. Schleberger, "Laser cleaning of exfoliated graphene," Mater Res Soc Symp Proc 1455 (2012), 10.1557/opl.2012.1196.

${ }^{23}$ A. Wucher, M. Wahl, and H. Oechsner, "The mass distribution of sputtered metal clusters," Nucl. Instrum. Methods Phys. Res. B 83, 73-78 (1993).

${ }^{24}$ L. Breuer, P. Ernst, M. Herder, F. Meinerzhagen, M. Bender, D. Severin, and A. Wucher, "Mass spectrometric investigation of material sputtered under swift heavy ion bombardment," Nucl. Instrum. Methods Phys. Res. B 435, 101-110 (2018).

${ }^{25}$ M. Herder, P. Ernst, L. Skopinski, B. Weidtmann, M. Schleberger, and A. Wucher, "Ionization probability of sputtered indium atoms under impact of slow highly charged ions," J. Vac. Sci. Technol. B 38, 044003 (2020).

${ }^{26}$ A. V. Samartsev and A. Wucher, "Kinetic energy distributions of neutral In and $\mathrm{In}_{2}$ sputtered by polyatomic ion bombardment," Appl. Surf. Sci. 252, 6470-6473 (2006).

${ }^{27}$ A. Wucher, M. Wahl, and H. Oechsner, "Sputtered neutral silver clusters up to $\mathrm{Ag}_{18}$," Nucl. Instrum. Methods Phys. Res. B 82, 337-346 (1993)

${ }^{28}$ A. S. El-Said, R. A. Wilhelm, R. Heller, S. Facsko, C. Trautmann, and F. Aumayr, "Surface nanostructuring of $\mathrm{SrTiO}_{3}$ single crystals by slow highly charged ions and swift heavy ions," Nucl. Instrum. Methods Phys. Res. B 269, 1234-1237 (2011).

${ }^{29}$ C. Lemell, A. S. El-Said, W. Meissl, I. C. Gebeshuber, C. Trautmann, M. Toulemonde, J. Burgdörfer, and F. Aumayr, "On the nano-hillock formation induced by slow highly charged ions on insulator surfaces," SolidState Electronics 51, 1398-1404 (2007). 


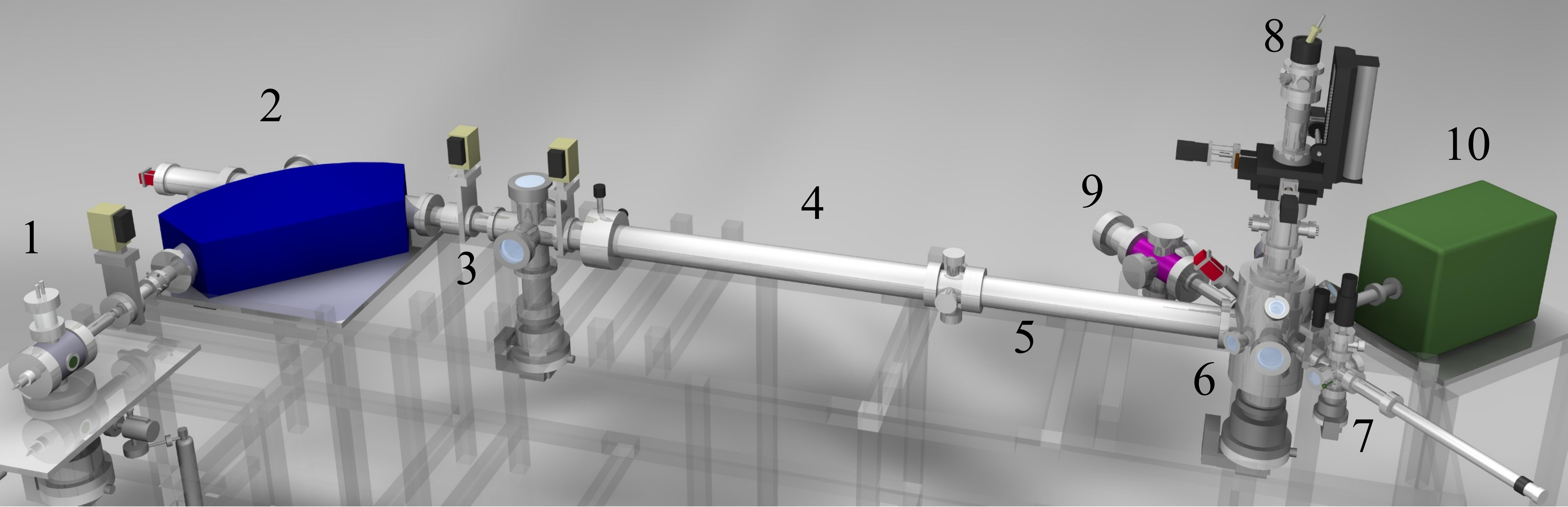




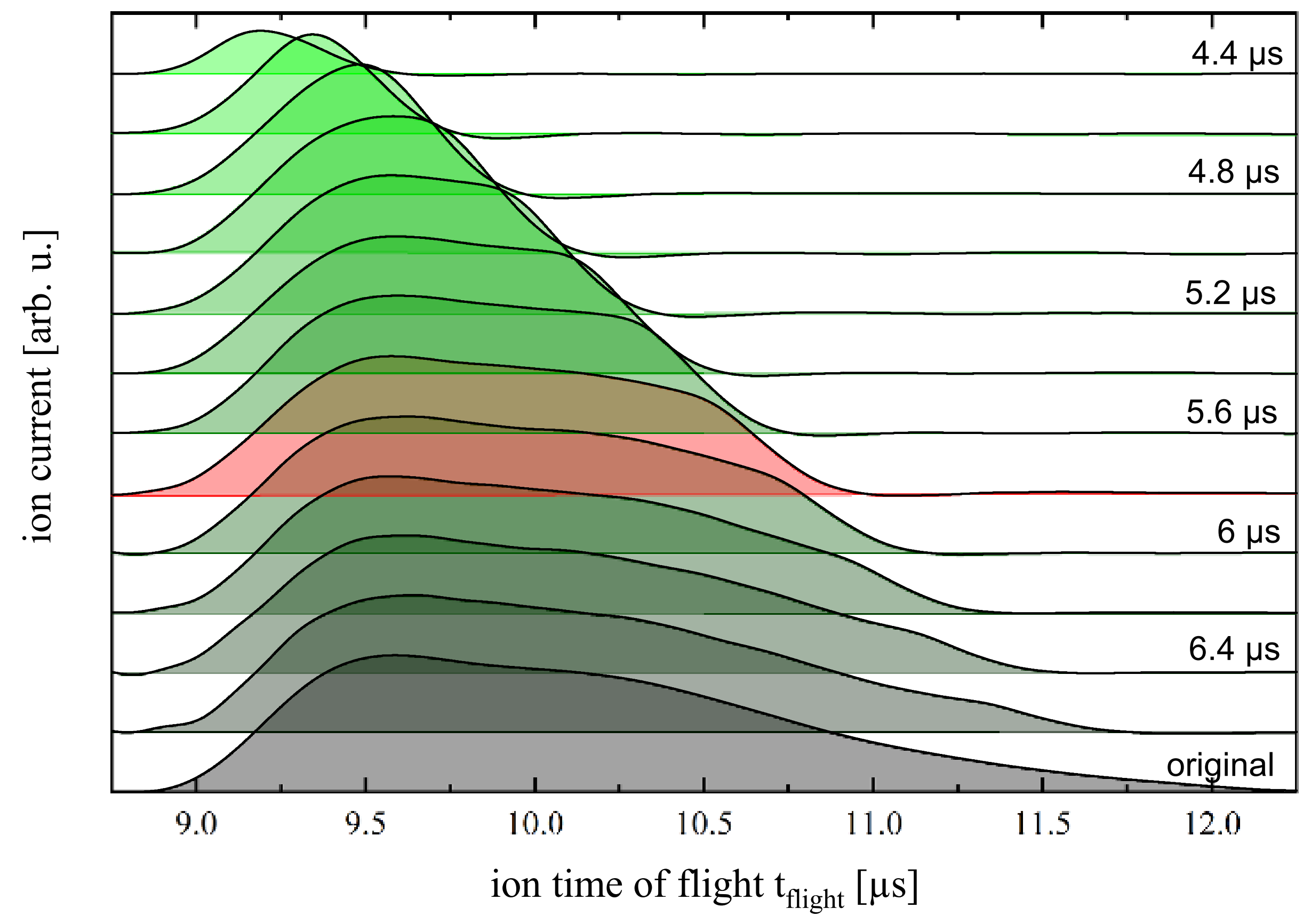


a)

c)

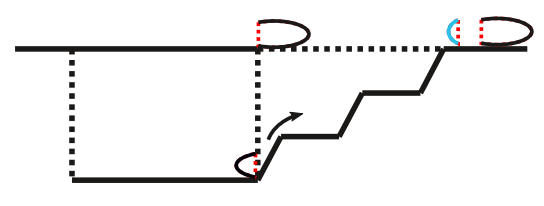

d)

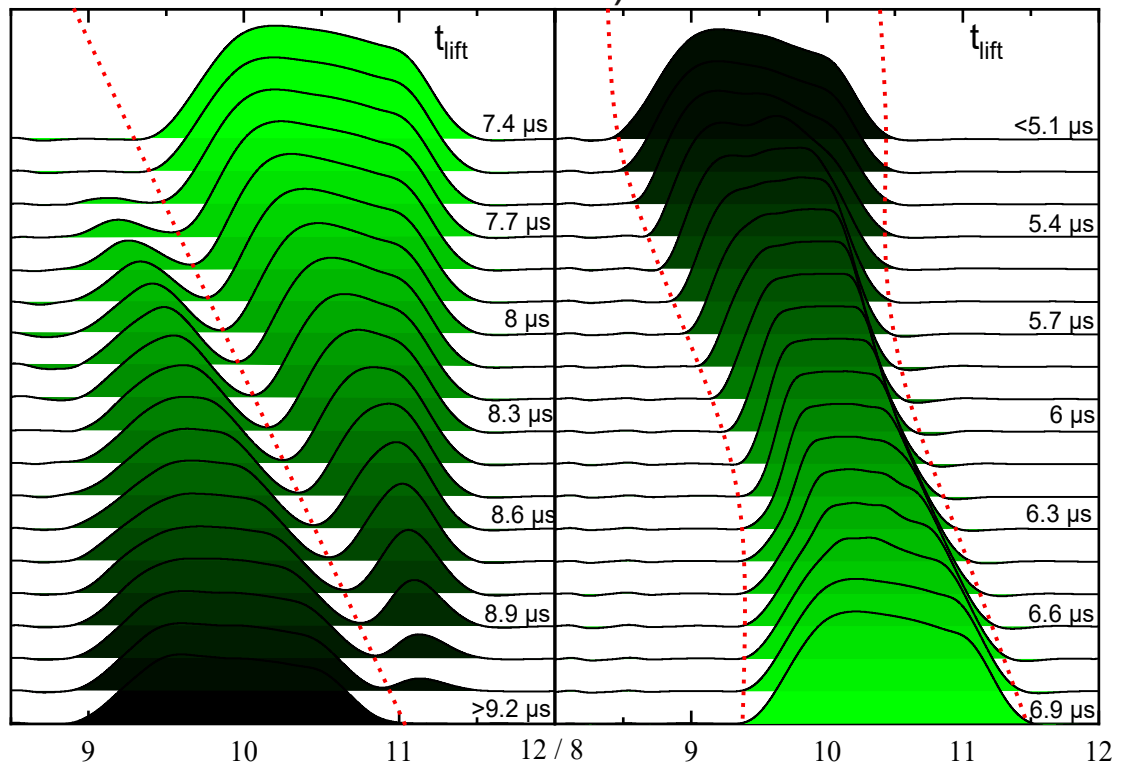

ion time of flight $\mathrm{t}_{\text {flight }}[\mu \mathrm{s}]$ 


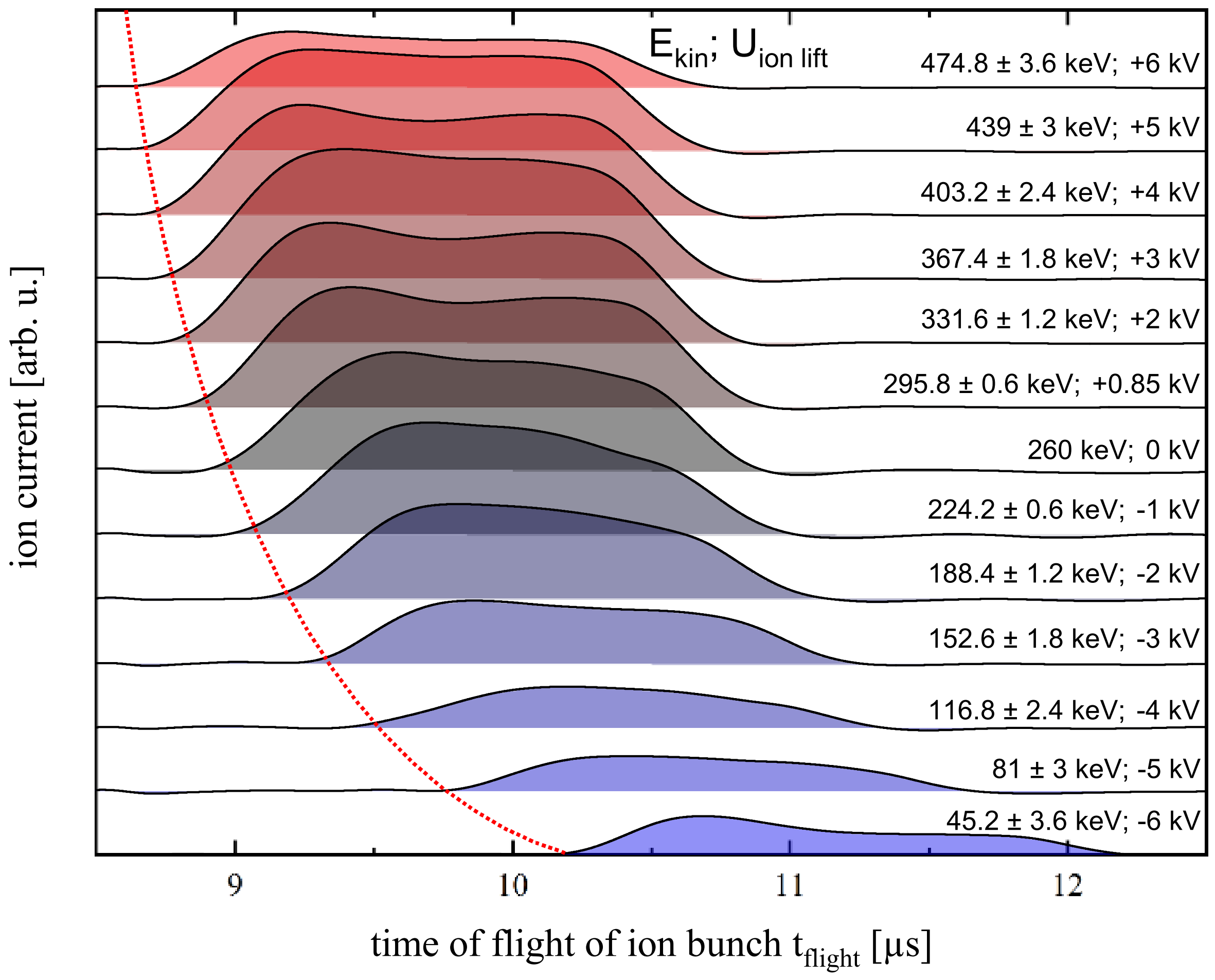




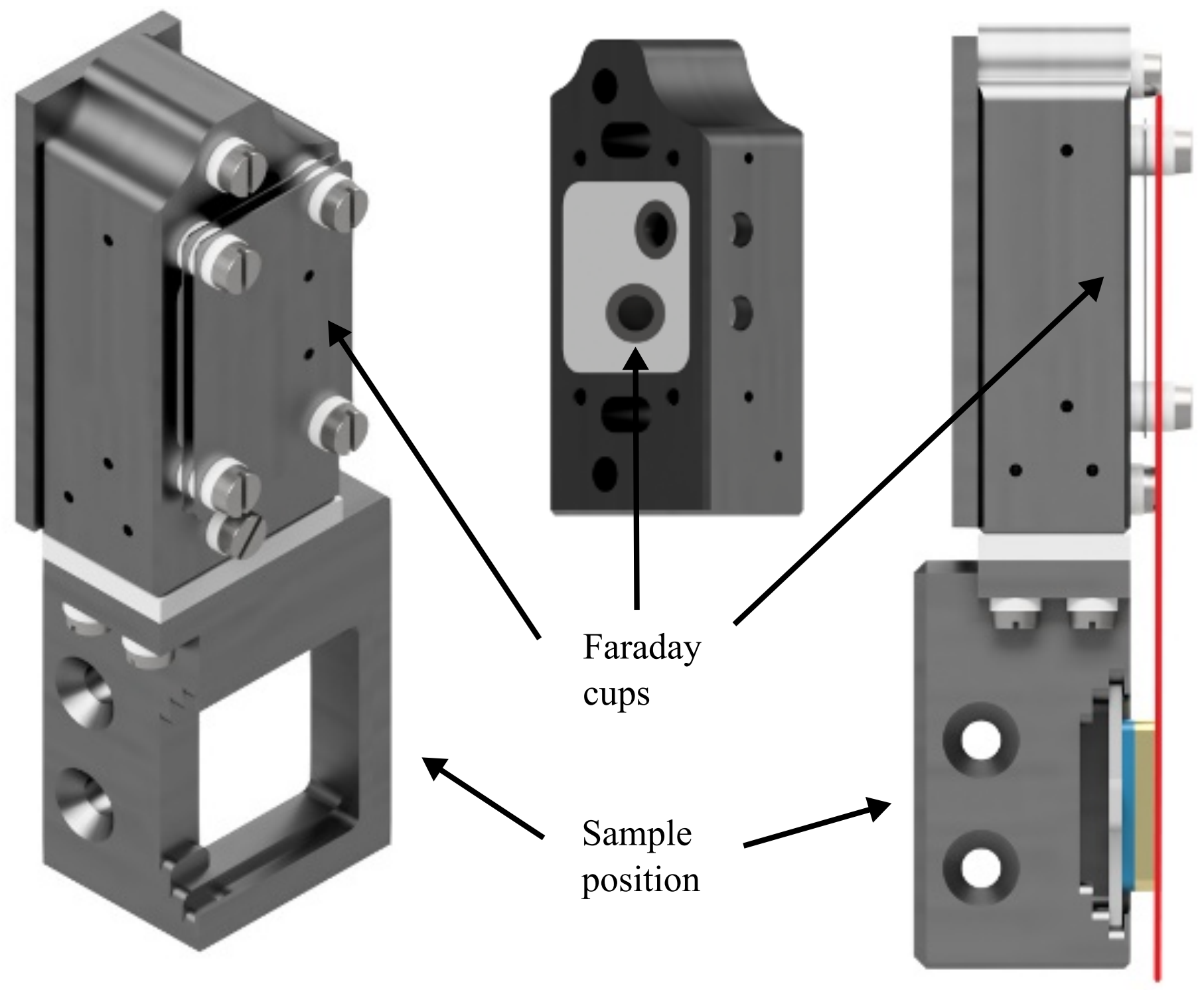




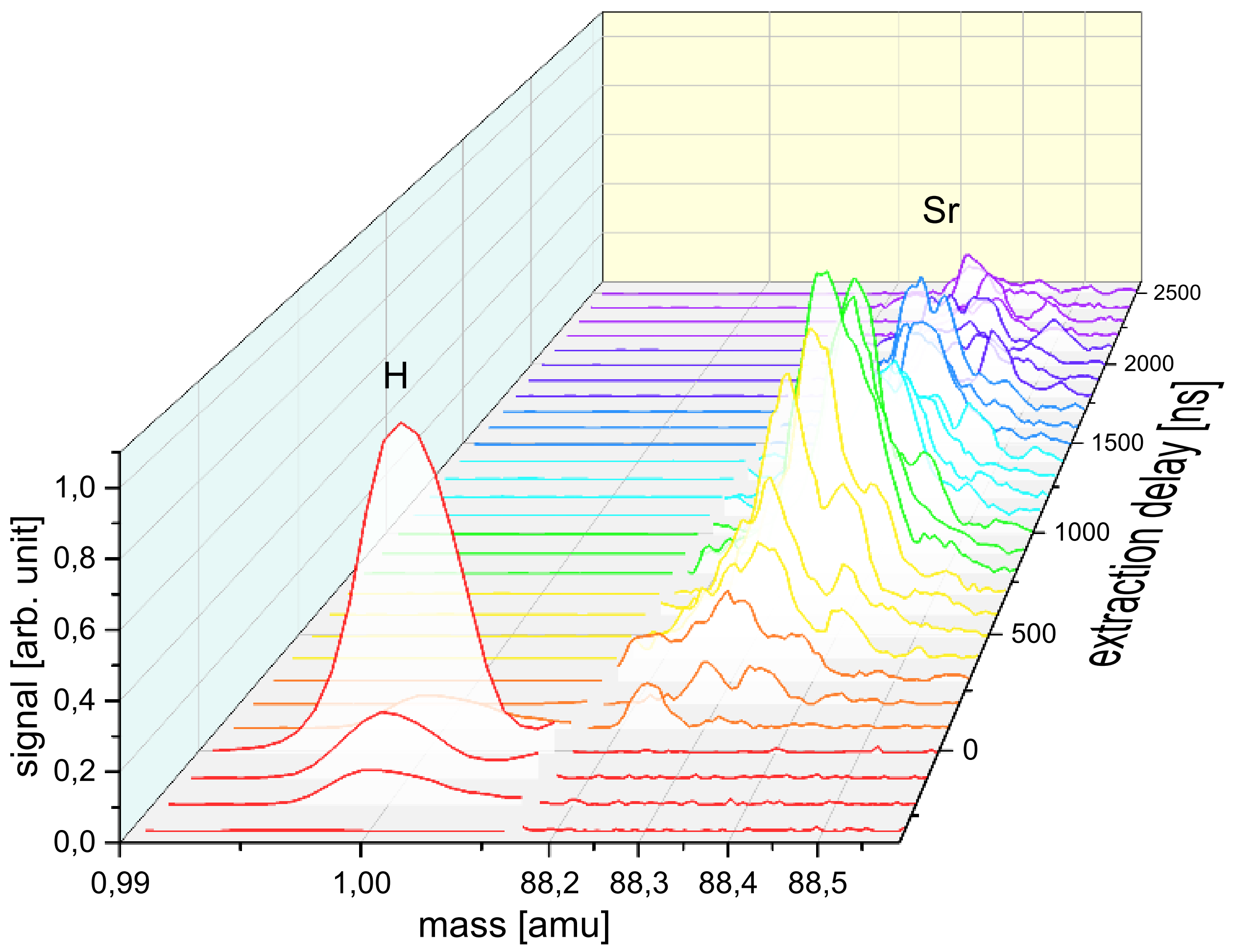




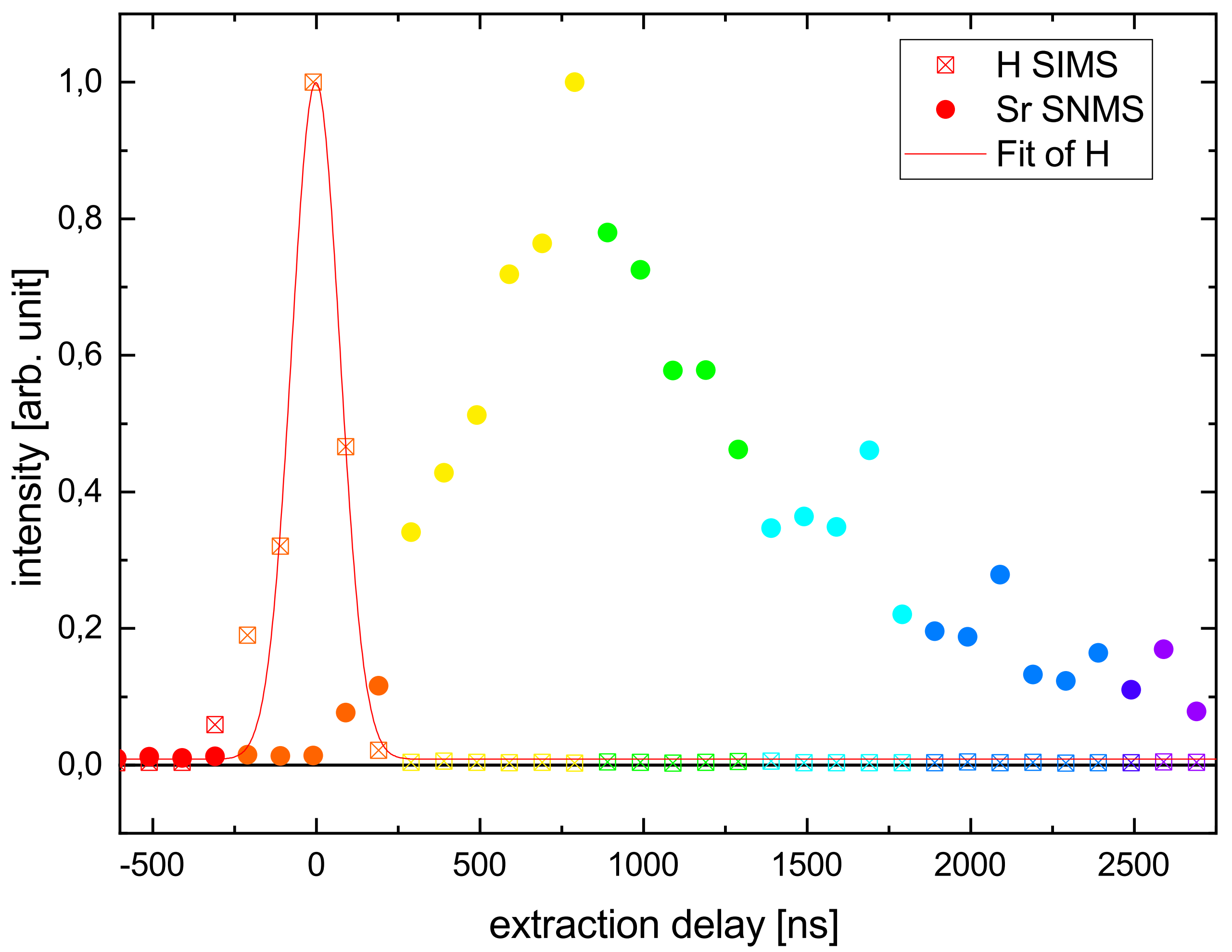




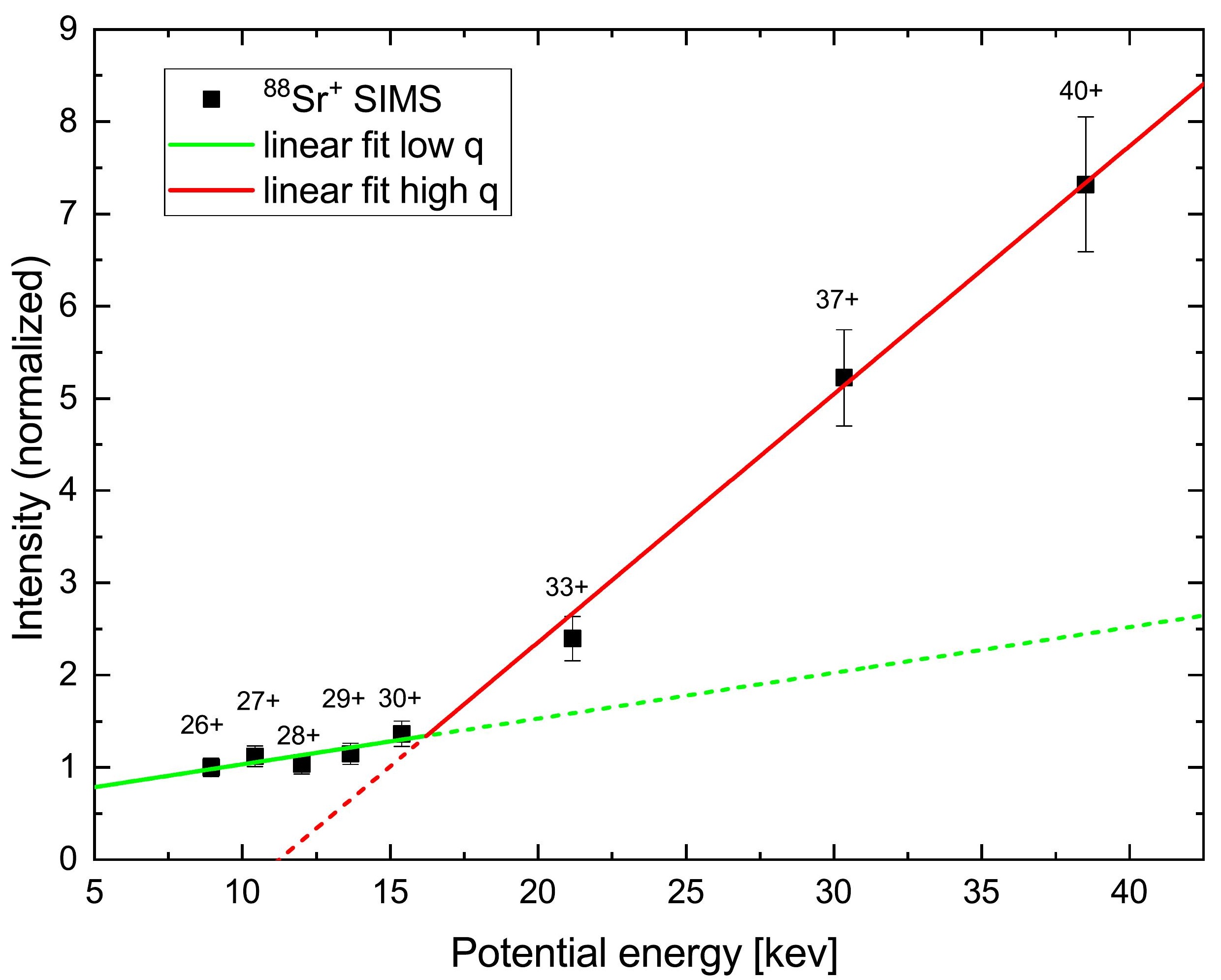




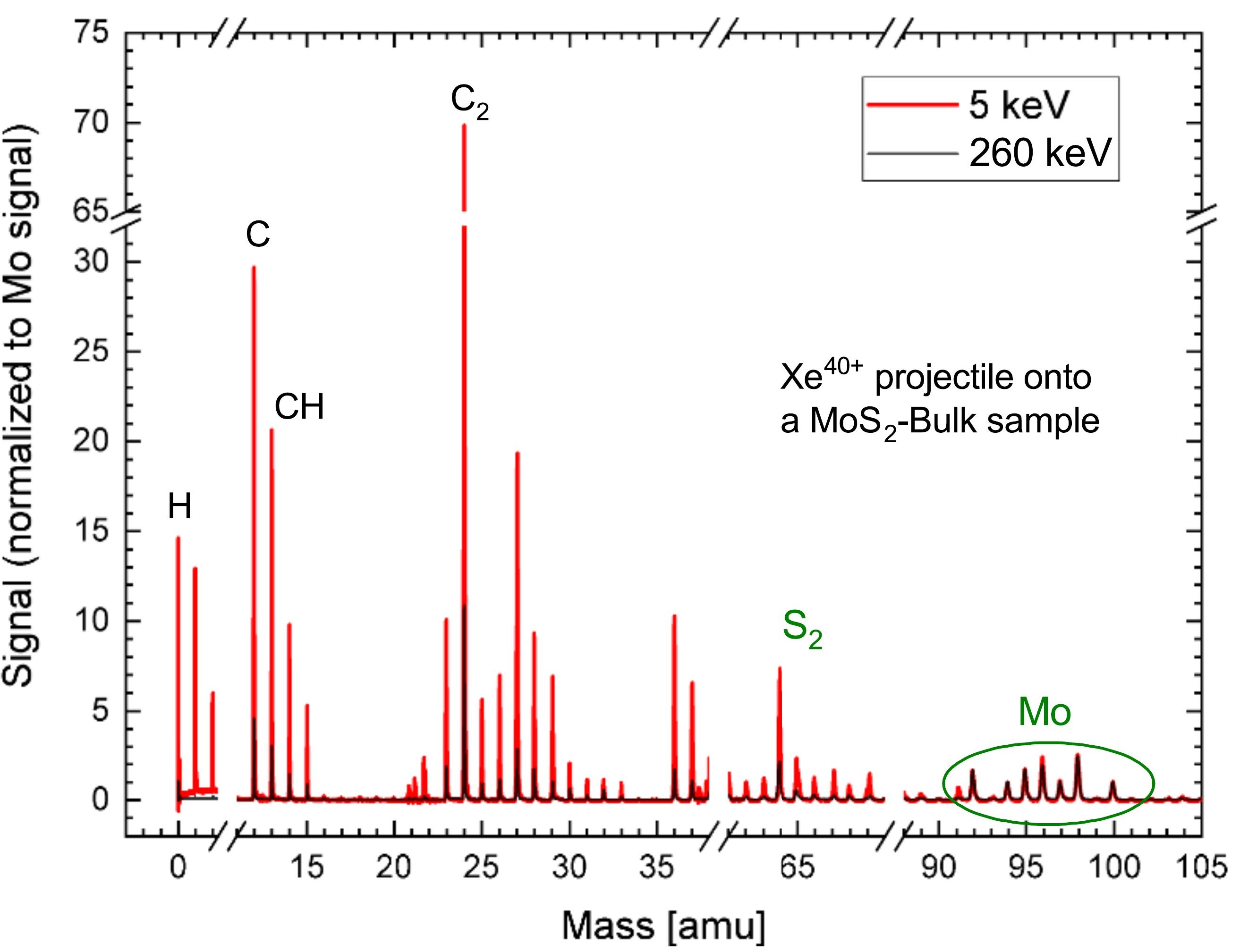



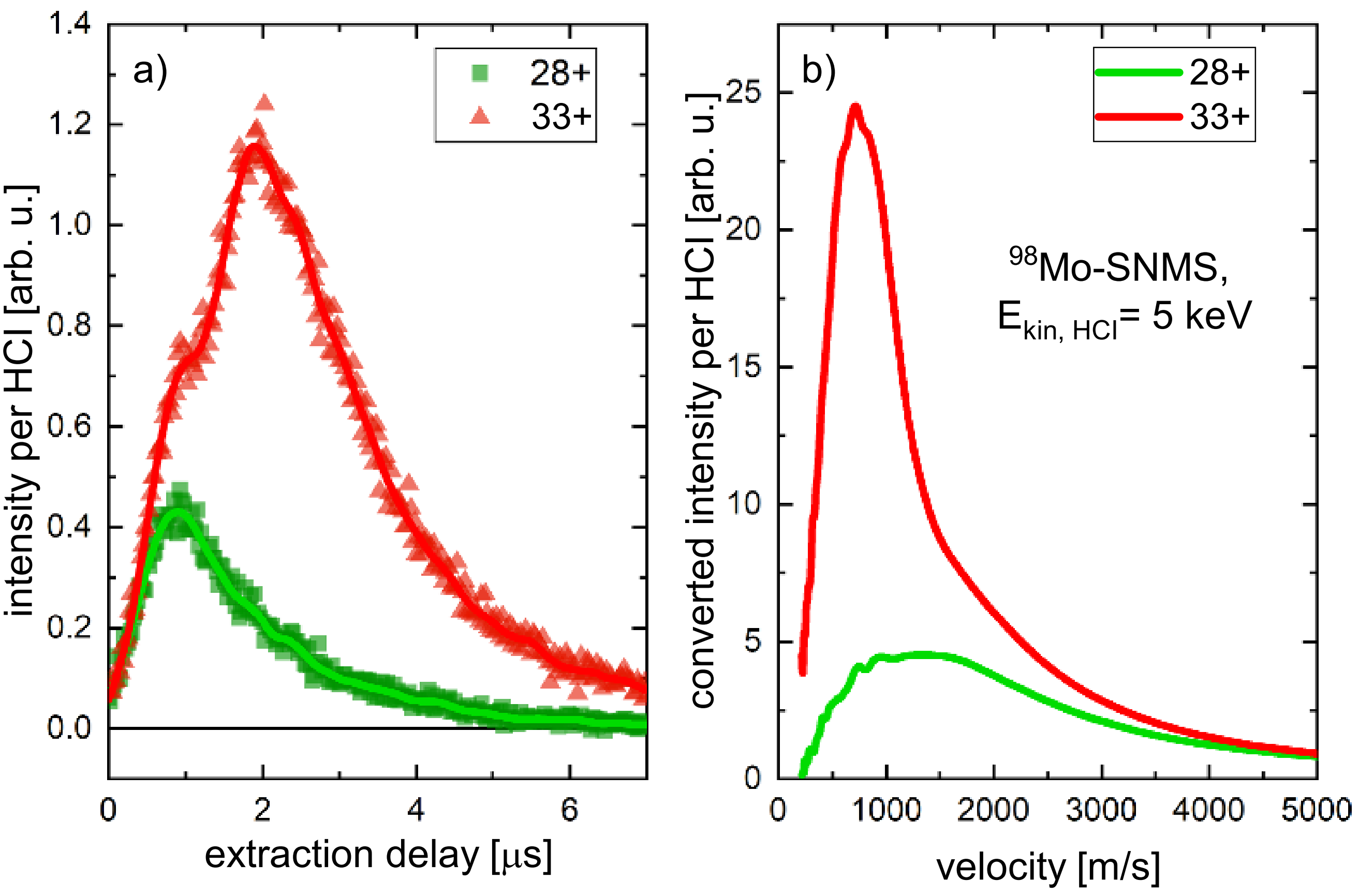